\title{
On SUSY GUTs with a degenerate Higgs mass matrix
}

\author{
F. Brümmer, ${ }^{a}$ S. Fichet, ${ }^{b}$ S. Kraml ${ }^{b}$ and R.K. Singh ${ }^{c}$ \\ ${ }^{a}$ Institute for Particle Physics Phenomenology, Durham University, \\ South Road, Durham DH1 3LE, U.K. \\ ${ }^{b}$ Laboratoire de Physique Subatomique et de Cosmologie, UJF Grenoble 1, CNRS/IN2P3, \\ 53 Avenue des Martyrs, F-38026 Grenoble, France \\ ${ }^{c}$ Institut für Theoretische Physik und Astronomie, Universität Würzburg, \\ Am Hubland, D-97074 Würzburg, Germany \\ E-mail: felix.bruemmer@durham.ac.uk, fichet@lpsc.in2p3.fr, \\ sabine.kraml@lpsc.in2p3.fr, singh@physik.uni-wuerzburg.de
}

ABSTRaCT: Certain supersymmetric grand unified models predict that the coefficients of the quadratic terms in the MSSM Higgs potential, $m_{1,2}^{2} \equiv m_{H_{1,2}}^{2}+|\mu|^{2}$ and $m_{3}^{2} \equiv B_{\mu}$, should be degenerate at the grand-unified scale. We discuss some examples for such models, and we analyse the implications of this peculiar condition of a GUT-scale degenerate Higgs mass matrix for low-scale MSSM phenomenology. To this end we explore the parameter space which is consistent with existing experimental constraints by means of a Markov Chain Monte Carlo analysis.

Keywords: Supersymmetric Standard Model, Beyond Standard Model, GUT

ARXiv EPRINT: 1007.0321 


\section{Contents}

1 Introduction 1

2 Models 3

2.1 Gauge-Higgs unification in 5d 3

2.2 Holographic GUTs 6

$\begin{array}{llr}3 & \text { Soft term patterns } & 8\end{array}$

4 Markov Chain Monte Carlo analysis $\quad 15$

$\begin{array}{lll}4.1 & \text { Results for universal soft terms } & 17\end{array}$

4.2 Results for vanishing 1st/2nd generation soft terms 20

5 Conclusions $\quad 24$

\section{Introduction}

The quadratic part of the Higgs potential in the Minimal Supersymmetric Standard Model (MSSM) may be written as

$$
V=m_{1}^{2}\left|H_{1}\right|^{2}+m_{2}^{2}\left|H_{2}\right|^{2}+m_{3}^{2}\left(H_{2} H_{1}+\text { h.c. }\right) \text {. }
$$

Here $m_{1,2}^{2}$ are given in terms of the soft SUSY breaking masses $m_{H_{1,2}}^{2}$ and the supersymmetric Higgsino mass $\mu$ as $m_{1,2}^{2}=m_{H_{1,2}}^{2}+|\mu|^{2}$. The soft parameter $m_{3}^{2}$ is often also called $B_{\mu}$; the phases are defined such that $m_{3}^{2}>0$ at the electroweak scale. $H_{1,2}$ are the lowest components of the down-type and up-type Higgs superfields (which we will also denote by $H_{1,2}$ ).

In models of gravity-mediated SUSY breaking, the Higgs mass parameters are usually generated at the GUT scale or the Planck scale by some mechanism which breaks supersymmetry. (Even the $\mu$ parameter, although it preserves supersymmetry, should be generated by the SUSY-breaking mechanism in order to explain why it is of the same order of magnitude as the other Higgs mass parameters.) They should then be evolved down to the electroweak scale according to their renormalization group equations. At the scale where the Higgs potential is minimized, they should satisfy the well-known inequalities

$$
m_{1}^{2} m_{2}^{2}-m_{3}^{4}<0, \quad m_{1}^{2}+m_{2}^{2}-2 m_{3}^{2}>0 .
$$

The first inequality ensures that electroweak symmetry is broken, and the second one guarantees that the Higgs potential is bounded from below even in those directions in field space where the quartic potential vanishes.

An interesting property of certain UV-scale models is the relation

$$
m_{1}^{2}=m_{2}^{2}= \pm m_{3}^{2}
$$


holding at the UV scale, where the SUSY-breaking terms are generated. This is the defining relation for the class of models we are interested in, models with a degenerate Higgs mass matrix (DHMM). It is typically encountered in models where both MSSM Higgs doublets originate from a single chiral adjoint $\Phi$ of the GUT group $G$, by a decomposition into Standard Model representations according to

$$
\begin{aligned}
\operatorname{Ad}(G) & \rightarrow(\mathbf{1}, \mathbf{2})_{-1 / 2} \oplus(\mathbf{1}, \mathbf{2})_{1 / 2} \oplus \ldots \\
\Phi & \rightarrow \quad H_{1} \oplus H_{2} \quad \oplus \ldots
\end{aligned}
$$

Suppose that there is some additional structure ensuring that, while the real components $\Phi^{\dagger}+\Phi$ can acquire a tree-level mass from SUSY breaking, the Higgs field components of the imaginary parts $\Phi^{\dagger}-\Phi$ remain massless. For instance, they could be the (pseudo)Goldstone bosons of a spontaneously broken (approximate) global symmetry $[1,2]$ or a mass term could be forbidden by higher-dimensional gauge invariance [3, 4]. Then the quadratic Higgs potential will be of the form

$$
V=m^{2}\left(\bar{H}_{1}+H_{2}\right)\left(H_{1}+\overline{H_{2}}\right) .
$$

Thus the relation eq. (1.3) obviously holds. A subtlety lies in the definition of the sign of $m_{3}^{2}$, which may need to be changed by a field redefinition $H_{1} \rightarrow-H_{1}$ to ensure that $m_{3}^{2}>0$ after running to the electroweak scale.

In realistic models in which the UV-scale equalities eq. (1.3) apply, renormalization group running should turn them into the IR-scale inequalities eq. (1.2). At the same time the correct Standard Model couplings and masses should be reproduced, and the spectrum of sparticle and Higgs masses should not be in conflict with experimental bounds. Whether this is possible for a given UV-scale model can only be found out by a numerical analysis. As discussed in $[3,4]$, it crucially depends on the parameters of the sfermion sector.

In a previous paper [4] a particular subclass of such models was investigated in detail with respect to their phenomenological prospects. More precisely, it was shown that in five-dimensional orbifold GUTs with gauge-Higgs unification and radion-mediated SUSY breaking, fully realistic MSSM spectra can be found. Such models are well motivated as anisotropic limits of heterotic string compactifications.

Since the class of DHMM models is in fact quite large and diverse, it is clearly of interest to study in detail under what conditions on the soft terms realistic MSSM vacua and a viable low-scale phenomenology can result, and what are the consequences for experiment. This is the purpose of this paper. In section 2 we first discuss classes of SUSY GUTs which imply DHMM boundary conditions. In section 3 we elaborate on RGE running and resulting soft term patterns. In section 4 we then present results of a Markov Chain Monte Carlo (MCMC) analysis of two variants of DHMM models. Finally section 5 contains our conclusions. 


\section{Models}

\subsection{Gauge-Higgs unification in $5 \mathrm{~d}$}

An interesting class of example models in which eq. (1.3) holds is given by $5 \mathrm{~d}$ orbifold GUTs with gauge-Higgs unification [3-6]. The fifth dimension is compactified on an interval whose size is given by the inverse GUT scale, and the GUT group is broken to the MSSM gauge group by boundary conditions. In terms of $4 \mathrm{~d}$ superfields, the $5 \mathrm{~d}$ gauge multiplet decomposes into a $4 \mathrm{~d}$ gauge superfield $V=-A_{\mu} \sigma^{\mu} \theta \bar{\theta}+\cdots(\mu=0, \ldots, 3)$ and a chiral adjoint $\Phi=\Sigma+i A_{5}+\cdots$ (where we have only written the leading terms in the $\theta$-expansion, and $V$ is in Wess-Zumino gauge). $\Phi$ contains the MSSM Higgs fields as in eq. (1.4). We can now choose a Kähler-Weyl frame such that the superpotential is independent of $\Phi$ when setting the MSSM matter fields to zero. By 5d gauge invariance, the Kähler potential can then only depend on the combination $\Phi^{\dagger}+\Phi$ on the quadratic level. The orthogonal combination $\Phi^{\dagger}-\Phi \sim A_{5}$, being a 5 d gauge field, is protected from getting a mass term.

This can be seen explicitly as follows: Suppose for the moment that the gauge symmetry were just $\mathrm{U}(() 1)$. The action is invariant under 5 d gauge transformations

$$
V \rightarrow V+\Lambda+\bar{\Lambda}, \quad \Phi \rightarrow \Phi+\partial_{5} \Lambda
$$

Here $\Lambda$ is an $x^{5}$-dependent chiral superfield. The inhomogeneous transformation behaviour of $\Phi$ shows that $\Phi$ cannot appear in the superpotential if $W$ is to be $5 \mathrm{~d}$ gauge-invariant, when setting the MSSM matter fields to zero. That is to say, it is always possible to shift harmonic terms from the Kähler potential into the superpotential, and any terms from $W$ into $K$, but a particularly natural formulation is one where $W$ and $K$ are separately 5 d gauge invariant. Consequently $\Phi$ cannot appear in $W$ (except in combination with other charged fields such as matter fields, which only give rise to Yukawa terms irrelevant to the Higgs potential, or light exotics, which we assume to be absent).

The crucial observation is now [4] that in this manifestly 5 d gauge-invariant formulation, the $\Phi$-dependent part of $K$ must be a function of the unique gauge-invariant combination

$$
\Phi+\bar{\Phi}-\partial_{5} V
$$

This combination reduces to $\Phi+\bar{\Phi}$ on the zero-mode level. In other words, if there is no linear term in $\Phi$, the low-energy effective Kähler potential for the zero modes has the structure

$$
K=\mathcal{K}\left(Z^{i}, \bar{Z}^{\bar{\jmath}}\right)+\tilde{\mathcal{Y}}\left(Z^{i}, \bar{Z}^{\bar{\jmath}}\right)(\Phi+\bar{\Phi})^{2}+\cdots
$$

Here the $Z^{i}$ denote collectively the compactification moduli and general hidden sector fields. $K$ cannot depend on the orthogonal combination $A_{5}=\operatorname{Im} \Phi=(\Phi-\bar{\Phi}) / 2$ essentially because the transformation law for $\operatorname{Im} \Phi$ involves $\operatorname{Im} \Lambda$, whereas the transformation law for $V$ only involves $\operatorname{Re} \Lambda$, and therefore the gauge variation of $\operatorname{Im} \Phi$ cannot be cancelled.

A similar structure is encountered in realistic models. The gauge symmetry should of course contain the Standard Model gauge group, and $\Phi$ should contain the MSSM Higgs 
superfields, so the abelian example is too simple. In a non-abelian model, the $5 \mathrm{~d}$ gauge transformations read

$$
e^{V} \rightarrow e^{\Lambda^{\dagger}} e^{V} e^{\Lambda}, \quad \Phi \rightarrow e^{-\Lambda}\left(\partial_{5}+\Phi\right) e^{\Lambda}
$$

Gauge-invariant operators involving $\Phi$ can be constructed from the covariant derivative $\nabla_{5}=\partial_{5}+\Phi[7]$. In particular the operator

$$
-e^{-V} \nabla_{5} e^{V}=\Phi+\Phi^{\dagger}-\partial_{5} V+(\text { commutators })
$$

(where $\nabla_{5}$ acts on $e^{V}$ as $\nabla_{5} e^{V}=\partial_{5} e^{V}-\Phi^{\dagger} e^{V}-e^{V} \Phi$ ) is the appropriate non-abelian generalization of (2.2). Note that it is not gauge invariant by itself but transforms analogously to a field strength superfield:

$$
e^{-V} \nabla_{5} e^{V} \rightarrow e^{-\Lambda} e^{-V} \nabla_{5} e^{V} e^{\Lambda}
$$

The lowest-order gauge invariant operator one can construct is in fact [7]

$$
\operatorname{tr}\left(e^{-V} \nabla_{5} e^{V}\right)^{2}=\operatorname{tr}\left(\Phi+\Phi^{\dagger}\right)^{2}+(\text { terms involving } V)
$$

since $\operatorname{tr}\left(e^{-V} \nabla_{5} e^{V}\right)$ vanishes identically, as can be seen from eq. (2.5).

As in the abelian case, any $V$-independent terms cannot depend on the orthogonal combination $\Phi-\Phi^{\dagger}$ since it transforms as

$$
\Phi-\Phi^{\dagger} \rightarrow e^{-\Lambda} \Phi e^{\Lambda}-\text { h.c. }+\partial_{5}\left(\Lambda-\Lambda^{\dagger}\right)
$$

while the gauge field transforms as

$$
V \rightarrow V+\Lambda+\Lambda^{\dagger}+(\text { terms involving } V)
$$

Therefore there is no function of $V$ whose gauge variation can cancel the inhomogeneous piece in eq. (2.8).

We conclude that again $W$ is $\Phi$-independent, and that $K$ has the structure

$$
K=\mathcal{K}\left(Z^{i}, \bar{Z}^{\bar{\jmath}}\right)+\tilde{\mathcal{Y}}\left(Z^{i}, \bar{Z}^{\bar{\jmath}}\right) \operatorname{tr}\left(\Phi+\Phi^{\dagger}\right)^{2}+\cdots
$$

The resulting quadratic Lagrangian for the zero modes of $\Phi$ can then be written as

$$
\mathcal{L}_{\text {quad }}=\int d^{4} \theta \bar{\varphi} \varphi \mathcal{Y}\left(Z^{i}, \bar{Z}^{\bar{\jmath}}\right)\left(\bar{H}_{1}+H_{2}\right)\left(H_{1}+\overline{H_{2}}\right)
$$

Here $\varphi=1+F^{\varphi} \theta^{2}$ is the conformal compensator of 4 d supergravity, a non-dynamical chiral superfield whose $F$-term is the scalar auxiliary field of the 4 d gravitational multiplet; its expectation value parameterises SUSY breaking in the $4 \mathrm{~d}$ gravitational background. A non-vanishing $F^{\varphi}$ or non-vanishing $F^{i}$ will give rise to an effective Higgs mass matrix 


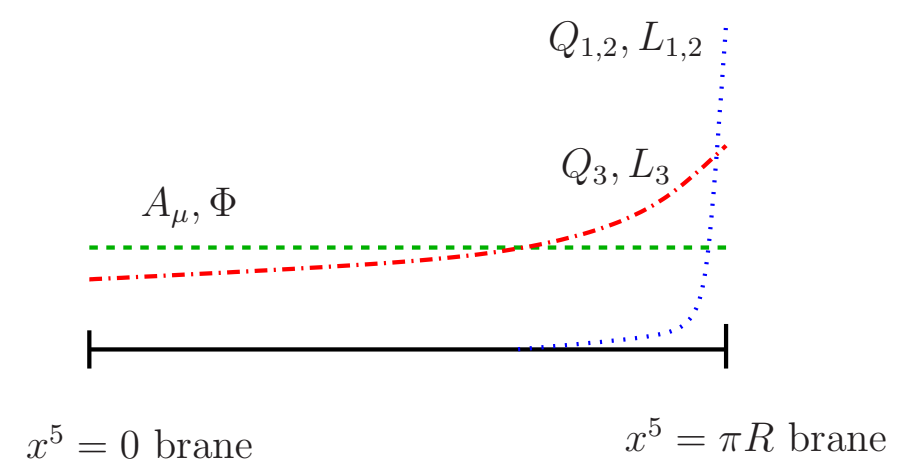

Figure 1. An unwarped model with gauge-Higgs unification. The field $\Phi$ containing the Higgs fields and the $4 \mathrm{~d}$ gauge fields (green dashed curve) are bulk fields with a flat profile. Third generation matter fields (red dot-dashed curve) are slightly localized towards the $x^{5}=\pi R$ brane. Matter fields of the first two generations (blue dotted curve) are effectively confined to the brane.

satisfying the relations eq. (1.3), with mass parameters ${ }^{1}$

$$
\begin{aligned}
m_{H_{1}}^{2} & =m_{H_{2}}^{2}=-F^{i} \bar{F}^{\bar{\jmath}} \frac{\partial^{2}}{\partial Z^{i} \partial \bar{Z}^{\bar{\jmath}}} \log \mathcal{Y}, \\
\pm \mu & =\bar{F}^{\bar{\varphi}}+\bar{F}^{\bar{\imath}} \frac{\partial}{\partial \bar{Z}^{\bar{l}}} \log \mathcal{Y}, \\
\pm B_{\mu} & =\left|F^{\varphi}+F^{i} \frac{\partial}{\partial Z^{i}} \log \mathcal{Y}\right|^{2}-F^{i} \bar{F}^{\bar{\jmath}} \frac{\partial^{2}}{\partial Z^{i} \partial Z^{\bar{\jmath}}} \log \mathcal{Y} .
\end{aligned}
$$

The relations eq. (1.3) are a direct consequence of 5 d gauge symmetry, which is however not a symmetry of the $4 \mathrm{~d}$ effective theory (but instead mixes the KK modes). Eq. (1.3) is therefore valid at the compactification scale, but it will be modified by radiative corrections below this scale. This is indeed necessary in order for the conditions eq. (1.2) to be satisfied as strict inequalities, after renormalization group running to the electroweak scale.

Models of this kind can be augmented by bulk hypermultiplets whose zero modes give the MSSM matter fields, and with brane fields with appropriate superpotentials to decouple unwanted exotics [5]. 4d Yukawa couplings are obtained from 5d gauge couplings, with their precise values controlled by the localization properties of the zero-mode wave functions, which in turn are tunable through $5 \mathrm{~d}$ mass terms. The full model has the massless spectrum of the MSSM. With the additional assumption that SUSY breaking mediation is dominated by the $F$-term of the radion modulus, it can give rise to realistic phenomenology [4]. See figure 1 for a sketch of this kind of model.

By similar arguments as above, the relations eq. (1.3) also apply in a large class of heterotic string orbifold models [8-11] with gauge-Higgs unification, if their moduli space

\footnotetext{
${ }^{1}$ As already alluded to, the signs of $\mu$ and $m_{3}^{2}$ can be simultaneously flipped by a superfield redefinition $H_{1} \rightarrow-H_{1}$ (together with a corresponding redefinition of the down-type matter superfields to keep the Yukawa couplings intact). We adopt the usual convention that $m_{3}^{2}>0$ at the weak scale. Since the sign of $m_{3}^{2}$ can change during its renormalization group evolution, this implies that the proper high-scale sign in eq. (2.12) is only fixed after specifying the rest of the model and tracking its RG running. The relation eq. (1.3) thus can be written as $m_{1}^{2}=m_{2}^{2}=\epsilon_{H} m_{3}^{2}$ with $\epsilon_{H}= \pm 1$ to be determined accordingly.
} 


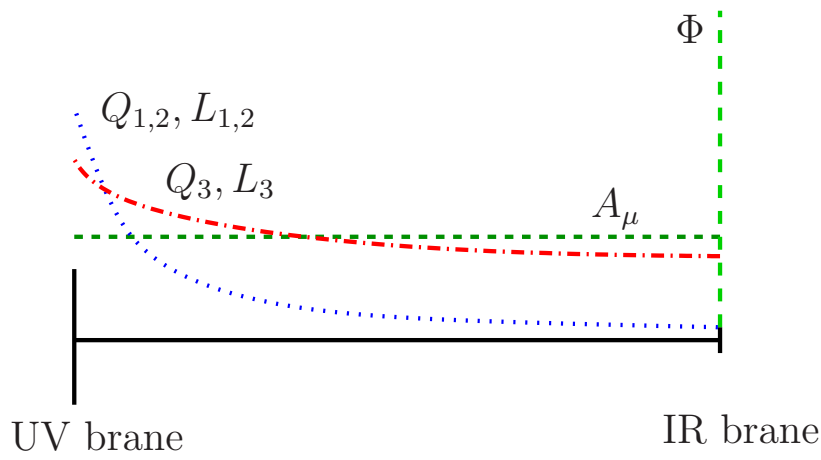

Figure 2. A warped holographic GUT model: $\Phi$ (light green dashed curve) is an IR brane field. Matter fields are localized towards the UV brane, only slightly so for the third generation but more pronounced for the first two generations.

admits a corresponding $5 \mathrm{~d}$ orbifold GUT limit (for details see e.g. [4, 12]). This is regardless of whether or not this anisotripic limit is actually realized at the point where the moduli are stabilized. The structure enforced by higher-dimensional gauge invariance persists independently.

Radion mediation (corresponding to "modulus domination" in the above-mentioned string models) is a simple and elegant possibility to parameterise SUSY breaking in such models. However, in general there may be other contributions, in particular from branelocalized fields (see e.g. [3]). This allows for more general patterns of soft masses than the ones considered in [4], providing a strong motivation for the more general parameter space scan which we perform in this paper.

\subsection{Holographic GUTs}

A somewhat different example is the holographic GUT model of Nomura, Poland and Tweedie [13]. This model may be described in the "gravity picture" as a 5d theory on a slice of $\mathrm{AdS}_{5}$ space between two 4d branes, a Planck brane (or UV brane) and a GUT brane (or IR brane). The bulk gauge symmetry is $\mathrm{SU}(6)$. The MSSM Higgs fields arise from a GUT-brane chiral superfield $\Phi$ in the adjoint. ${ }^{2} \mathrm{SU}(6)$ is spontaneously broken to $\mathrm{SU}(4) \times \mathrm{SU}(2) \times \mathrm{U}(1)$ by the $\Phi$ superpotential on the GUT brane, and explicitly broken to $\mathrm{SU}(5) \times \mathrm{U}(1)$ by boundary conditions on the Planck brane. In the $4 \mathrm{~d}$ effective theory the gauge group is then given by the intersection of $\mathrm{SU}(4) \times \mathrm{SU}(2) \times \mathrm{U}(1)$ and $\mathrm{SU}(5) \times \mathrm{U}(1)$ in $\mathrm{SU}(6)$, which is the Standard Model gauge group apart from an extra U(1). Matter fields arise from $5 \mathrm{~d}$ bulk fields. This model is sketched in figure 2 .

Regarding $\mathrm{SU}(6)$ as a spontaneously broken global symmetry of the $4 \mathrm{~d}$ theory, the Higgs fields are massless in the absence of SUSY breaking because their imaginary parts are Goldstone bosons associated with the breaking to $\mathrm{SU}(4) \times \mathrm{SU}(2) \times \mathrm{U}(1)$, and their real parts are then protected by SUSY. SU(6) is also broken explicitly by boundary conditions on the Planck brane (or on the level of the $4 \mathrm{~d}$ theory by gauging only its $\mathrm{SU}(5) \times \mathrm{U}(1)$ subgroup), and therefore the Higgs fields are merely pseudo-Goldstone bosons. However,

\footnotetext{
${ }^{2}$ In [13] this chiral adjoint is denoted by $\Sigma$.
} 
this explicit breaking gives tree-level masses to only 12 out of the 16 Goldstone modes. In other words, if $\mathrm{SU}(5) \times \mathrm{U}(1) \subset \mathrm{SU}(6)$ is gauged, only 12 of the Goldstone bosons will be eaten by the Higgs mechanism, corresponding to the 12 broken generators in $\mathrm{SU}(5) \times \mathrm{U}(1) \rightarrow \mathrm{SU}(3) \times \mathrm{SU}(2) \times \mathrm{U}(1) \times \mathrm{U}(1)$. The remaining four Goldstone modes and their complex partners form two massless weak doublets which are identified with the MSSM Higgs fields. (For some earlier work along these lines, see [1, 2].)

Provided that the SUSY breaking mechanism respects this symmetry breaking pattern, it will lead to eq. (1.3) because once more the fields from the combination $\Phi^{\dagger}+\Phi$ can pick up a tree-level mass term, while the (pseudo-) Goldstone bosons in $\Phi^{\dagger}-\Phi$ remain massless. Since the SU(6) symmetry is explicitly broken, radiative corrections can again lift the relations of eq. (1.3) below the SUSY breaking scale, as required for phenomenology.

Localizing the Higgs fields on one of the branes rather than in the bulk naturally allows for the possibility that they are "sequestered" from the supersymmetry breaking fields $Z^{i}$, which could be confined to the other brane. Writing the effective quadratic Higgs Lagrangian as in eq. (2.11), this means that there exists a frame in which

$$
\frac{\partial \mathcal{Y}}{\partial Z^{i}} F^{i}=0
$$

In that case any tree-level Higgs mass terms should arise purely from gravitational effects. We are parametrising these by the chiral compensator $\varphi$. Note that this type of scenario includes the case of radion mediation, i.e. taking the dominant source for SUSY breaking to be the $F$-term of the radion modulus, since one can always redefine $\varphi$ such that the radion does not couple to IR brane fields. It is well-known that $F^{\varphi}$ alone cannot induce scalar soft masses classically (in accord with eq. (2.12)), with the leading contribution to $m_{H_{1}}^{2}$ and $m_{H_{2}}^{2}$ coming from anomaly mediation and thus suppressed by a loop factor $[14,15]$. $F^{\varphi}$ will however give rise to a $\mu$ and a $B_{\mu}$ term at tree-level. One thus obtains a special case of eqs. (1.3) and (2.12):

$$
|\mu| \approx\left|F^{\varphi}\right|, \quad\left|B_{\mu}\right| \approx\left|F^{\varphi}\right|^{2}, \quad\left|m_{H_{i}}^{2}\right| \ll\left|F^{\varphi}\right|^{2} .
$$

Moreover, in the holographic GUT model with SUSY breaking on the UV brane there is no reason to expect the matter soft terms for the first two generations to be suppressed. The wave functions of the first and second generation have in fact similar to slightly larger overlaps with the UV brane, as compared with those of the third generation. This is because we are generating the Yukawa hierarchy from hierarchical wave function values on the IR brane, as depicted in figure 2.

Needless to say, this model also allows for supersymmetry breaking on the IR brane. In that case Higgs soft masses will generically be induced by direct contact interactions, and the ordering of sfermion masses will roughly correspond to the Yukawa hierarchy. In fact in general one can have a variety of different contributions to SUSY breaking mediation from brane-localized fields, as well as from the radion and from $\varphi$. 


\section{Soft term patterns}

In the common public SUSY spectrum generators, $\mu$ and $B_{\mu}$ at the GUT scale are computed according to their renormalization group equations from their IR-scale values, which in turn are calculated from $m_{Z}$ and the given $\tan \beta$. Here we use SOFTSUSY [16]. Following [4], we implement the Higgs mass relation eq. (1.3) by iteratively adjusting the Higgs soft masses,

$$
m_{H_{1}}^{2}=m_{H_{2}}^{2} \rightarrow \epsilon_{H} B_{\mu}-|\mu|^{2}
$$

at $M_{X}=M_{\mathrm{GUT}}$. Here $\epsilon_{H}= \pm 1$ takes care of the sign ambiguity in $B_{\mu}$ which we mentioned in the previous section. Throughout this analysis we assume gaugino mass unification, $M_{1}=M_{2}=M_{3} \equiv M_{1 / 2}$ at $M_{\mathrm{GUT}}$. The free parameters in our study are thus $M_{1 / 2}\left(M_{\mathrm{GUT}}\right), \tan \beta\left(M_{Z}\right)$, the two signs $\epsilon_{H}$ and $\operatorname{sign}(\mu)$, and the sfermion soft terms at $M_{\mathrm{GUT}}$. We take the latter to be flavour-diagonal.

Whether EWSB and a viable phenomenology can be obtained strongly depends on the sfermion soft terms. Two limiting cases are of particular interest:

- assuming a common sfermion mass $m_{0}$ and a common trilinear coupling $A_{0}$. This makes the DHMM models a subclass of non-universal Higgs mass models (see e.g. [17] and references therein) with $m_{H_{1}}^{2}=m_{H_{2}}^{2}$ ("NUHM1" in the terminology of [18]);

- no-scale boundary conditions for the first and second generation, $m_{0}(1,2)=$ $A_{0}(1,2) \equiv 0$, but allowing for arbitrary soft terms in the third generation.

The first case may be considered as representative for a generic scenario with all sfermion soft terms of the same order of magnitude, whereas the second case represents models with hierarchical soft terms reflecting the Yukawa hierarchy. As we have seen in section 2, both these scenarios are well motivated from the model-building point of view. Moreover, it is interesting to investigate whether the stronger condition of eq. (2.14), $m_{H_{1,2}}^{2} \rightarrow 0$ and $|\mu|^{2} \rightarrow\left|B_{\mu}\right|$, can be realized.

It turns out that the following patterns emerge in the soft terms:

1. in almost all of the admissible regions of parameter space, $\epsilon_{H}$ corresponds to the GUT-scale sign of $B_{\mu}$;

2. for $\operatorname{sign}(\mu)=+1, B_{\mu}$ has almost always the same sign as $A_{t}$ at the GUT scale (and the opposite one if $\operatorname{sign}(\mu)=-1$ );

3. for $\operatorname{sign}(\mu)=+1$, the stricter relation

$$
m_{H_{1}}^{2}=m_{H_{2}}^{2}=0, \quad \epsilon_{H} B_{\mu}=|\mu|^{2} \quad \text { at } M_{\mathrm{GUT}}
$$

can only be satisfied with $\epsilon_{H}=+1$.

These observations can be explained by a close inspection of the relevant RGEs, as we will now detail. 
To start with, it is useful to recall the dominant contributions to the one-loop RG evolution of the stop trilinear $A_{t}$. These involve $A_{t}$ itself and the gluino mass:

$$
16 \pi^{2} \frac{d}{d t} A_{t}=12\left|y_{t}\right|^{2} A_{t}+\frac{32}{3} g_{3}^{2} M_{3}+\cdots
$$

The large gluino contribution will drive $A_{t}$ to a large negative value towards "late times" (i.e. towards the low energy scale), until it is compensated for by the first term in eq. (3.3).

Now concerning point 1., if $\operatorname{sign}\left(B_{\mu}\right)$ does not match with $\epsilon_{H}$ at the GUT scale, this implies that the GUT-scale $m_{1}^{2}$ and $m_{2}^{2}$ are negative. The running of $m_{1}^{2}$ and $m_{2}^{2}$ is almost exclusively due to the running of the soft masses $m_{H_{1}}^{2}$ and $m_{H_{2}}^{2}$, since $\mu$ is approximately constant. The dominant terms in the one-loop RGE for the up-type Higgs soft mass-squared are

$$
16 \pi^{2} \frac{d}{d t} m_{H_{2}}^{2}=6\left|y_{t}\right|^{2}\left(\left|A_{t}\right|^{2}+m_{H_{2}}^{2}+m_{Q_{3}}^{2}+m_{U_{3}}^{2}\right)-6 g_{2}^{2}\left|M_{2}\right|^{2}+\cdots,
$$

where $m_{U_{3}}^{2}$ and $m_{Q_{3}}^{2}$ are the soft masses of the third generation up-type squarks and squark doublets respectively. In scenarios like the CMSSM, by scalar mass universality the terms in parentheses are typically positive. Thus $m_{2}^{2}$ is driven to lower values as the RG scale decreases, assisted also by the top Yukawa coupling and $\left|A_{t}\right|$ growing large. Eventually radiative electroweak symmetry breaking is triggered. Most of the DHMM parameter space also has this property. There is only a tiny region with initially small $A_{t}$, very large negative $m_{H_{1,2}}^{2}$, small or negative squark soft masses-squared, and sizeable $M_{1 / 2}$, in which $m_{2}^{2}$ runs up significantly at first. In that case it can be driven to positive values even if it starts out negative, and electroweak symmetry breaking can be triggered later when the $A_{t}$ contribution in eq. (3.4) dominates and when also the squark masses have grown positive.

Concerning point 2. above, note that $B_{\mu}$ at the low scale should be somewhat small compared to the typical soft masses. This is in order to satisfy the electroweak symmetry breaking conditions eq. (1.2), and in particular to have at least moderately large $\tan \beta$ ( $\tan \beta \gtrsim 5$ say). Let us for now assume positive $\mu$. The one-loop RGE of $B_{\mu}$ is dominated by the $A_{t}$ and gaugino contributions:

$$
16 \pi^{2} \frac{d}{d t} B_{\mu}=6 \mu A_{t}\left|y_{t}\right|^{2}+6 \mu M_{2} g_{2}^{2}+\cdots
$$

The gaugino contribution tends to dominate the RG evolution of $B_{\mu}$ at scales close to the GUT scale, driving $B_{\mu}$ down. However, eventually $A_{t}$ itself will run large and negative because of the gluino contribution to eq. (3.3). Far in the IR it will thus primarily drive the $B_{\mu}$ evolution, causing $B_{\mu}$ to run up instead. For sizeable and positive initial GUT-scale $A_{t}$, this latter effect will be less important. But for small or even negative GUT-scale values, $A_{t}$ will quickly evolve towards large negative values, and thus dominate over the gaugino term in eq. (3.5).

We conclude that positive starting values for the GUT-scale $A_{t}$ are preferred if $B_{\mu}>0$ (in which case $B_{\mu}$ should mainly run down towards the electroweak scale, to end up small) and small or negative values are preferred if $B_{\mu}<0$ (in which case it should mainly run up, to end up positive). For negative $\mu$, the signs are reversed. These correlations are 

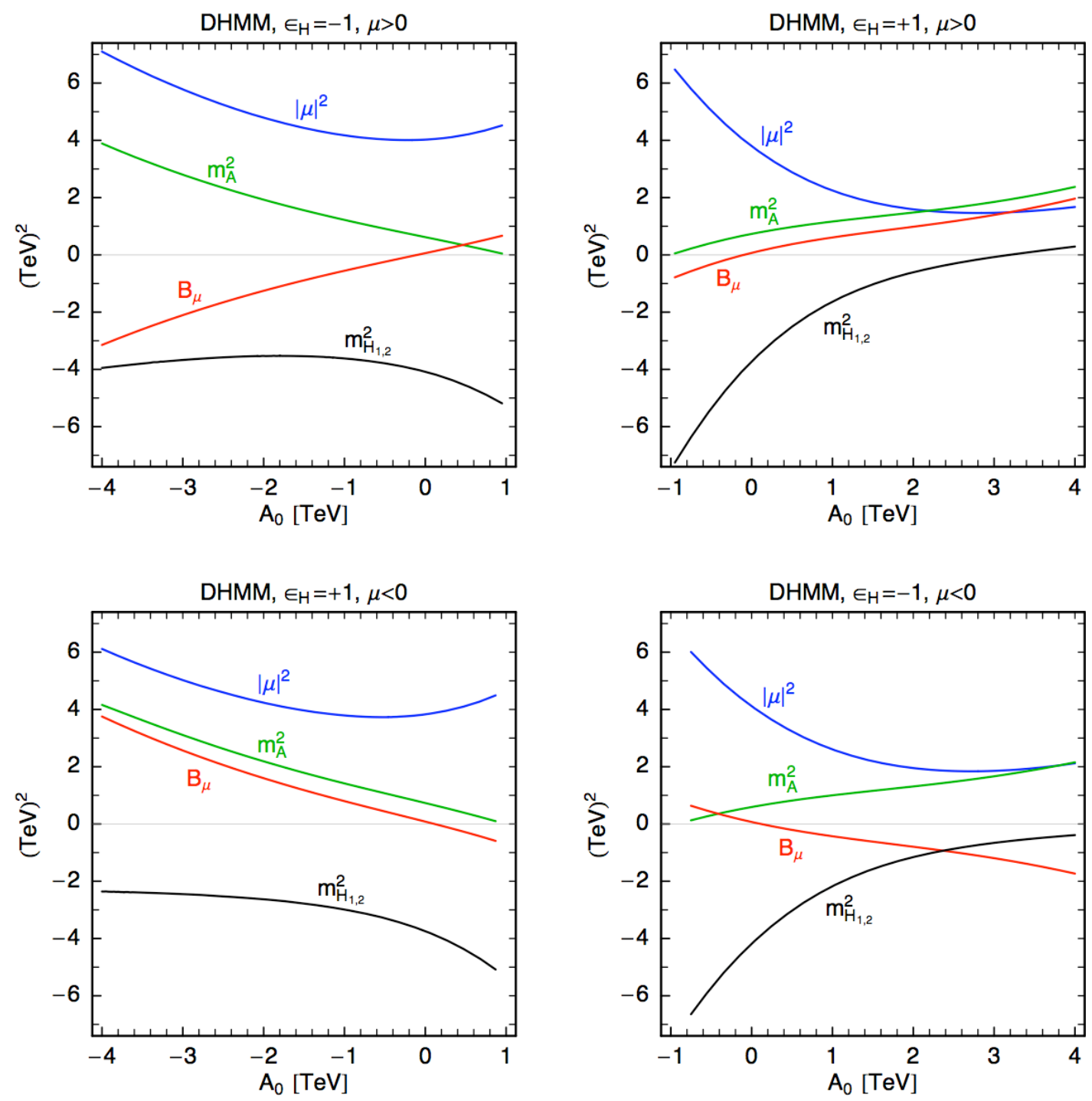

Figure 3. A set of slices through the four branches of DHMM parameter space with $M_{1 / 2}=1 \mathrm{TeV}$, $\tan \beta=10$, and $m_{0}=500 \mathrm{GeV}$. For the panels on the left, regions of larger $A_{0}$ do not lead to electroweak symmetry breaking, as can be seen from the pseudoscalar Higgs mass $m_{A}^{2}$ approaching zero. The same is true for regions of smaller $A_{0}$ for the panels on the right. The upper panels show, as explained in the text, that for $\operatorname{sign}(\mu)=+1$ the GUT-scale sign of $A_{t}$ is equal to the GUT-scale sign of $B_{\mu}$ in almost all of the allowed regions. The lower panels show that this correlation is reversed if $\operatorname{sign}(\mu)=-1$. As also explained in the text, $m_{H_{1,2}}^{2}=0$ is only possible if $\epsilon_{H}=+1$ and $\operatorname{sign}(\mu)=+1$ (top right panel). Finally note that there is a tiny slice of the allowed parameter space where $\epsilon_{H}$ does not correspond to $\operatorname{sign}\left(B_{\mu}\right)$ at the GUT scale. In this region $\left|A_{t}\right|$ is small and $m_{H_{2}}^{2}$ is large and negative as expected. 

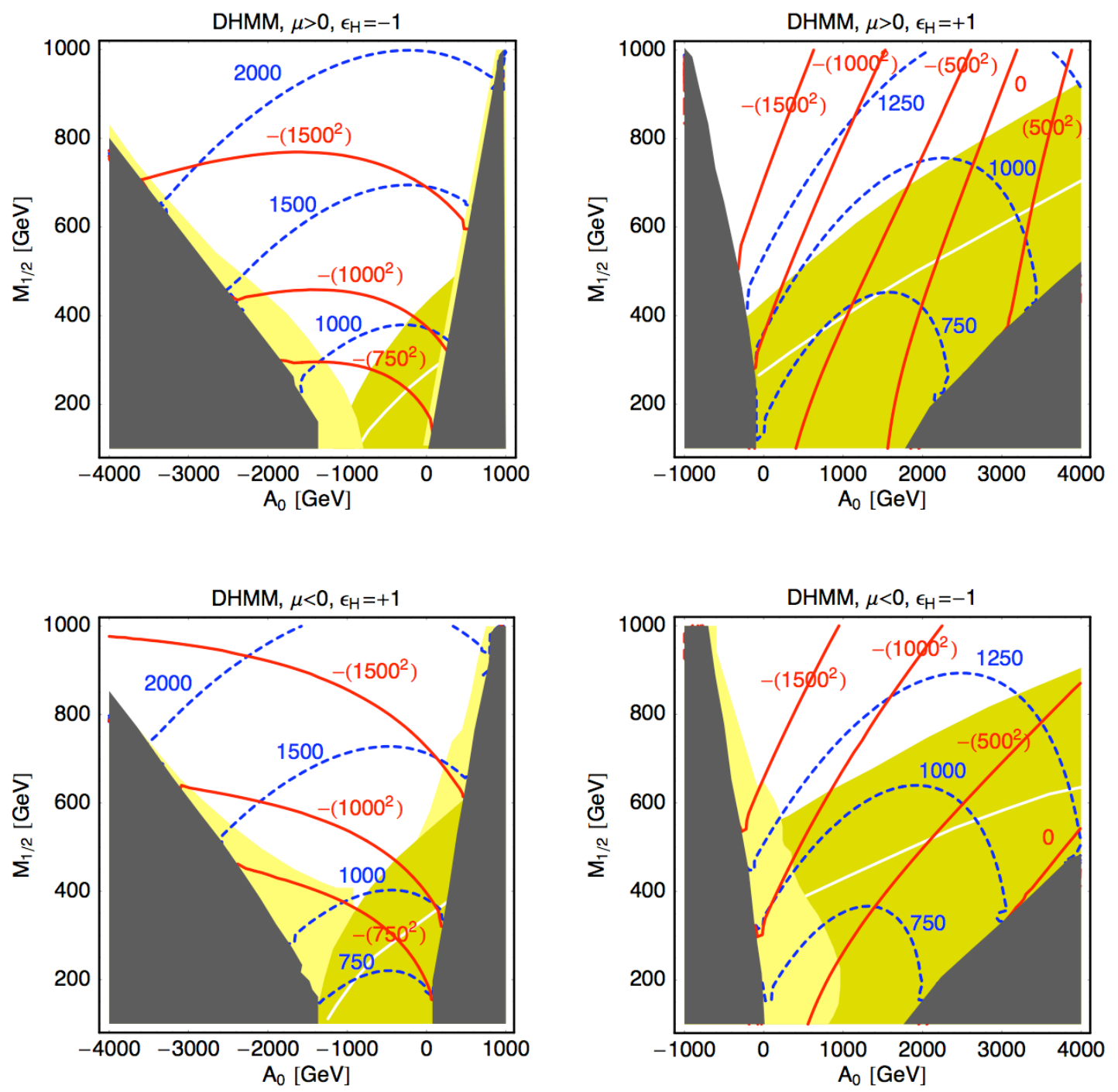

Figure 4. Contours of constant $m_{H_{1,2}}^{2}$ (full red lines) and $|\mu|$ (dashed blue lines) at $M_{\mathrm{GUT}}$ in the $M_{1 / 2}$ versus $A_{0}$ plane, for $\tan \beta=10$ and $m_{0}=500 \mathrm{GeV}$. Grey regions do not lead to electroweak symmetry breaking, light yellow regions are excluded by $b \rightarrow s \gamma$ (at $2 \sigma)$, and dark yellow regions have $m_{h}<114 \mathrm{GeV}$, with the white lines indicating $m_{h}=111 \mathrm{GeV}$.

illustrated in figure 3 with the help of some slices through the parameter space, at fixed sfermion and gaugino masses, fixed $\tan \beta$, and universal trilinears.

Our choice for the soft masses in figure 3 may seem rather high, but as can be seen from figure 4 , satisfying the LEP Higgs bound requires fairly large $M_{1 / 2}$, the more so the larger $A_{0}$ is.

Finally let us come to point 3.: The $A_{t}$ parameter also enters the $m_{H_{2}}^{2}$ RGE eq. (3.4). A large $\left|A_{t}\right|$ will accelerate the decrease of $m_{H_{2}}^{2}$ when running down from the GUT scale, which is of course a particularly severe effect if $A_{t}$ starts out negative, i.e. if $\epsilon_{H}=-\operatorname{sign}(\mu)$. 
Then $m_{H_{2}}^{2}$ will run negative too quickly, unless there is some other contribution to counterbalance the effect of $A_{t}$. Large gaugino masses could provide such a contribution, but they would again slow down the $B_{\mu}$ evolution, as is evident from eq. (3.5). If we allow for non-vanishing Higgs soft masses-squared, they can in particular be negative and thus counteract the $A_{t}$ effect in eq. (3.4). However, in the case that $m_{H_{1,2}}^{2}$ is constrained to vanish, $A_{t}$ should be positive at the GUT scale to minimize its effect on the $m_{H_{2}}^{2}$ running. In addition, small (or even negative) GUT-scale squark masses-squared are preferred to slow down the $m_{H_{2}}^{2}$ evolution. Indeed, in figures 3 and 4 the case $m_{H_{1,2}}^{2} \rightarrow 0$ occurs only for $\epsilon_{H}=\operatorname{sign}(\mu)$ and requires large positive $A_{0}$ to start with, leading to small negative $A_{t}$ at the EW scale.

As can also be seen from figure 4 , large positive $A_{0}$ leads to a tension with the direct search bound from LEP of $m_{h}>114.4 \mathrm{GeV}$ at $95 \%$ C.L. [19], even when taking into account a 2-3 GeV theoretical uncertainty [20] on the calculation of $m_{h}$ in the MSSM. This can be understood as follows: At lowest order, the light CP-even Higgs boson of the MSSM is at most as heavy as the $Z^{0}$ boson, $m_{h}^{2} \leq m_{Z}^{2} \cos ^{2} 2 \beta$. Radiative corrections have to lift $m_{h}$ above the LEP limit. The dominant effect is proportional to the fourth power of the top Yukawa coupling, $y_{t}^{4}$, and comes from an incomplete cancellation of top and stop loops. This increases $m_{h}$ approximately to

$$
m_{h}^{2} \lesssim m_{Z}^{2}+\frac{3 g^{2} m_{t}^{4}}{8 \pi^{2} m_{W}^{2}}\left[\ln \left(\frac{M_{S}^{2}}{m_{t}^{2}}\right)+\frac{X_{t}^{2}}{M_{S}^{2}}\left(1-\frac{X_{t}^{2}}{12 M_{S}^{2}}\right)\right]+\cdots,
$$

where

$$
M_{S}^{2} \equiv \frac{1}{2}\left(m_{\tilde{t}_{1}}^{2}+m_{\tilde{t}_{2}}^{2}\right), \quad X_{t} \equiv A_{t}-\mu \cot \beta .
$$

For large $\tan \beta$ and large $|\mu|$, also bottom and sbottom loops become important, giving an analogous contribution proportional to $y_{b}^{4}$. For details see, e.g., [21] and references therein. The logarithmic sensitivity to the average stop mass $M_{S}$ in eq. (3.6) suggests that heavy stops are preferred in order to render $m_{h}$ large enough. However, this sensitivity is rather mild, and the dependence on the stop mixing parameter $X_{t}$ can be at least as important. Indeed, $m_{h}$ initially increases with $\left|X_{t}\right|$ and reaches maximal values for $X_{t}= \pm \sqrt{6} M_{S}$; this is known as the 'maximal mixing' or $m_{h}^{\max }$ case, see again [21]. Therefore a large low-scale $\left|A_{t}\right|$, together with moderately large $\tan \beta$, is favoured to satisfy the LEP Higgs mass bound. This is exactly what we find in the right-hand side panels of figure 4: For too large starting values of $A_{t}$, the low-scale $\left|A_{t}\right|$ will be too small (recall that $A_{t}$ generically runs towards negative values) and the Higgs mass bound becomes important.

The DHMM model with universal sfermion soft terms is a special case of a NUHM model. Figure 5 shows for comparison the dependence of $m_{A}^{2}, \mu^{2}$ and $B_{\mu}$ in a general NUHM1 model with $m_{H_{1,2}}^{2}=0$. The other parameters are as in figure 3. The CMSSM limit with $m_{H_{1,2}}^{2}=m_{0}^{2}$ gives almost the same picture, the only difference being a slightly larger $m_{A}^{2}$ and slightly smaller $\mu^{2}$. Note that there is only one "DHMM point" in figure 3 (with meeting $|\mu|^{2}$ and $B_{\mu}$ curves), which occurs for $\mu>0$. Away from this point where the models coincide, DHMM has a much larger $|\mu|$ and smaller $m_{A}$ than NUHM1 (or the CMSSM), cf. figure 3. In particular, in the DHMM case $m_{A}$ becomes small for small $\left|A_{0}\right|$, and we can have $m_{A} \approx M_{1 / 2}$ even for small $\tan \beta$. This will be important later when we 


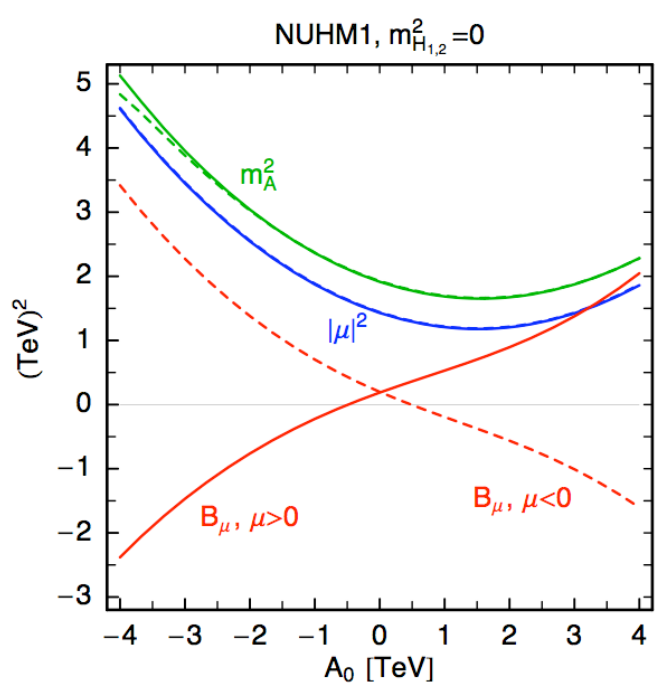

Figure 5. Higgs mass parameters in the MSSM with non-universal Higgs masses, for a universal gaugino mass $M_{1 / 2}=1 \mathrm{TeV}$, a universal sfermion mass $m_{0}=500 \mathrm{GeV}, \tan \beta=10$, and vanishing Higgs soft masses. Note that there is only a "DHMM point" (with meeting $|\mu|^{2}$ and $B_{\mu}$ curves) for $\mu>0$.

consider the neutralino relic density. At this stage we just remark that in figure $4 s$-channel annihilation through the Higgs funnel occurs for small $\left|A_{0}\right| \lesssim 100-300 \mathrm{GeV}$.

Let us now explore the consequences of non-universal third generation soft terms. This is interesting in particular for the gauge-Higgs unification models discussed in section 2.1, where we expect vanishing first and second generation soft masses, $m_{0}(1,2) \approx 0$. In this case we have roughly $m_{\tilde{e}_{R}}^{2} \approx\left(0.39 M_{1 / 2}\right)^{2}-0.052 S_{\mathrm{GUT}}$, and $m_{\tilde{e}_{L}}^{2} \approx\left(0.68 M_{1 / 2}\right)^{2}+$ $0.026 S_{\mathrm{GUT}}$, which has to be compared to $m_{\tilde{\chi}_{1}^{0}} \approx 0.43 M_{1 / 2}$. Here, $S_{\mathrm{GUT}}$ is the GUT-scale value of the hypercharge $S$ parameter,

$$
S=\left(m_{H_{2}}^{2}-m_{H_{1}}^{2}\right)+\operatorname{Tr}\left(m_{Q}^{2}-2 m_{U}^{2}+m_{D}^{2}+m_{R}^{2}-m_{L}^{2}\right) .
$$

We see that a non-zero and negative $S_{\mathrm{GUT}}$ of about $-\left(0.8 M_{1 / 2}\right)^{2}$ to $-\left(3.3 M_{1 / 2}\right)^{2}$ is necessary if one wants the neutralino to be the lightest SUSY particle (LSP). Since we have $m_{H_{1}}^{2}=m_{H_{2}}^{2}$ in DHMM, the way to ensure a neutralino LSP is non-universality of the third generation, as illustrated in figure $6 .^{3}$ We can see that taking a slice along $m_{U_{3}}=m_{Q_{3}}=m_{D_{3}}$, as we have done in the previous plots, indeed results in qualitatively similar patterns as the more general case in which the squark soft masses are split. On the other hand, negative soft masses-squared can give much smaller $m_{H_{1,2}}^{2}$ and $|\mu|$. This is of interest in particular for $\epsilon_{H}=\operatorname{sign}(\mu)=+1$, where one can achieve a mixed bino-higgsino LSP (see the dark matter discussion in the next section). Note moreover that in the r.h.s. plots of figure $6, m_{H_{1,2}}^{2}$ is very sensitive to $A_{0}$, while $\mu$ does not vary much when passing from $A_{0}=M_{1 / 2}$ to $A_{0}=3 M_{1 / 2}$. This is in accord with figure 3 .

\footnotetext{
${ }^{3}$ We use the notation $m_{U_{3}} \equiv m_{U_{3}}^{2} / \sqrt{\left|m_{U_{3}}^{2}\right|}$, so the sign of $m_{U_{3}}$ is actually that of $m_{U_{3}}^{2}$, and analogously for $m_{Q_{3}}$ etc.
} 

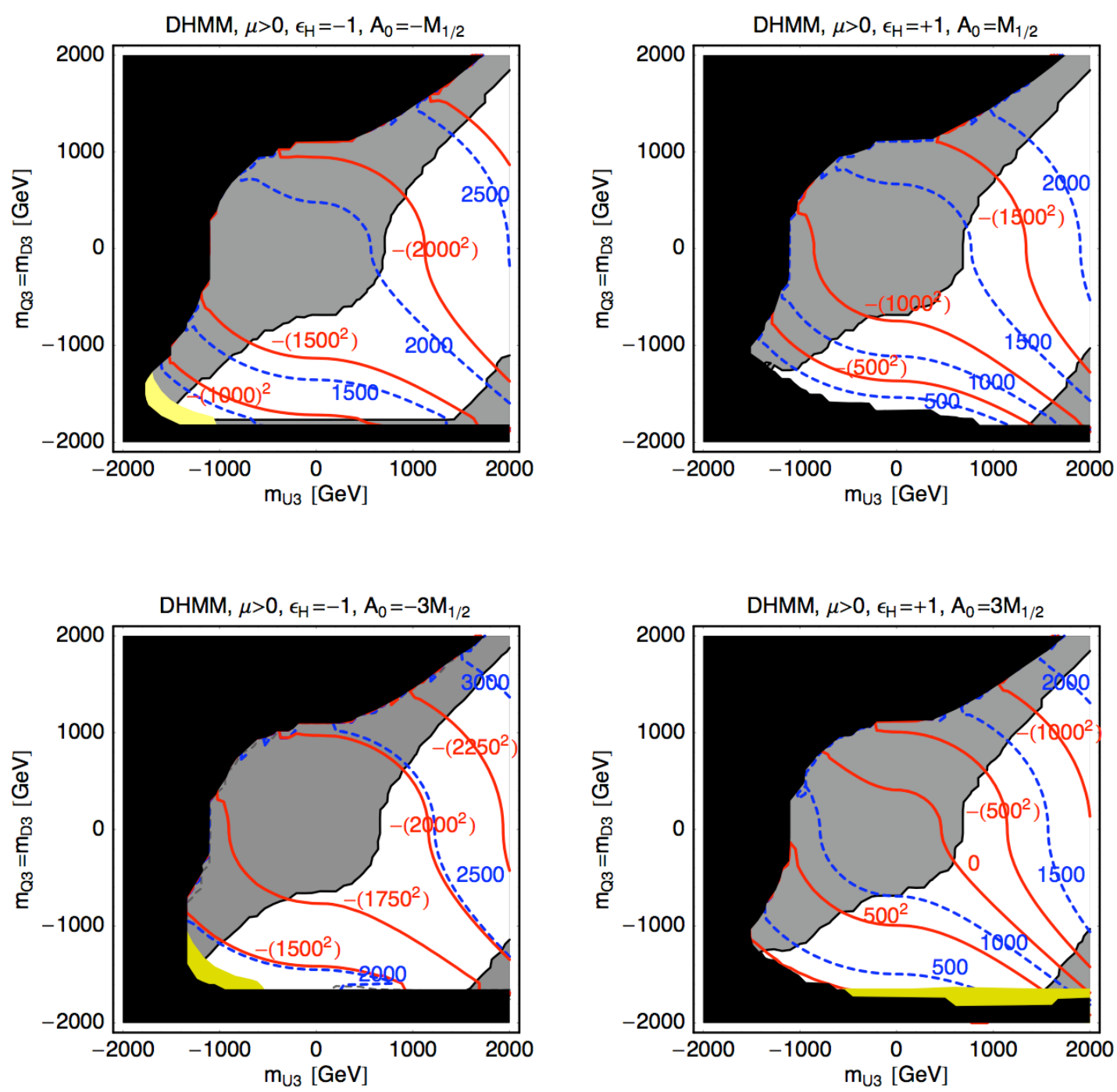

Figure 6. Contours of constant $m_{H_{1,2}}^{2}$ (full red lines) and $|\mu|$ (dashed blue lines) at $M_{\mathrm{GUT}}$ in the $m_{U_{3}}$ versus $m_{Q_{3}}$ plane, for $M_{1 / 2}=1 \mathrm{TeV}, \tan \beta=10$ and vanishing 1 st/2nd generation soft terms. Moreover, $\mu>0, \epsilon_{H}= \pm 1$, and $A_{0}= \pm M_{1 / 2}$ (upper row) and $A_{0}= \pm 3 M_{1 / 2}$ (lower row). Black regions do not lead to electroweak symmetry breaking, gray regions have a slepton LSP, and white regions a neutralino LSP; the light yellow stripe is excluded by $b \rightarrow s \gamma$ at $2 \sigma$, while the dark yellow stripes have $m_{h}<114 \mathrm{GeV} . \mu<0$ gives qualitatively very similar results.

We conclude this section with a few remarks on potentially dangerous tachyonic directions. In our analysis we have permitted tachyonic GUT-scale masses for both the Higgs and the sfermion fields. This is well-known to generally lead to charge- and colour-breaking minima in the potential, as well as to directions in field space which are unbounded from below (at tree-level and without higher-dimensional operators); see, for instance, [22].

While tachyonic scalar masses appear to rule out a large part of the parameter space at 
first sight, two points must be considered. First, a careful analysis is required in each case to determine if such dangerous vacua really are present. In particular, calculations using only the RG-improved tree-level potential may give unreliable results if the field VEVs are found to be vastly different from the renormalization scale, because of the presence of large logarithms in the loop corrections. Second, these vacua need not be dangerous even if they are present. If the tunnelling rate from our false vacuum is sufficiently small, our vacuum may well be effectively stable on cosmological timescales. It then depends on early-universe cosmology whether or not it is preferred for our universe. See [23] for a recent analysis of the CMSSM and of NUHM models in that context, and [24] for a recent analysis of the cosmological lifetime of related Higgs-exempt no-scale models.

A detailed investigation of charge- and colour-breaking minima is beyond the scope of this work. We therefore merely stress that we expect them to appear in large regions of parameter space, but depending on their lifetime and on the cosmological scenario, these regions may still be acceptable phenomenologically.

\section{Markov Chain Monte Carlo analysis}

So far we have only considered the constraints from $m_{h}$ and $b \rightarrow s \gamma$, and taken one- or two-dimensional slices through the parameter space. In order to take into account more constraints and in particular to find regions of parameter space where the neutralino LSP is a good dark matter candidate, we next perform a Markov Chain Monte Carlo scan of DHMM models. As above, we consider the two cases of (i) universal sfermion soft terms and (ii) vanishing first/second but non-universal third generation soft terms.

MCMC is an efficient method to probe a large-dimensional parameter space, and to gain information about it by using Bayesian statistics. The basic idea is to set up a random walk, starting at some parameter point and proposing a candidate next point at random nearby. This candidate point is then accepted or rejected at random, with an acceptance probability depending on its likelihood compared to the likelihood of the original point. Parameter points which are more likely to reproduce existing experimental data and constraints within errors have a greater probability of being accepted. If accepted, the new point is chosen as the starting point and the procedure is iterated. Otherwise it is repeated with the old starting point. A properly set-up ensemble of Markov chains should eventually fill out all the allowed parameter space, with a high density of points in those regions which are best compatible with existing measurements. In the sense of Bayesian statistics, the distributions of points are interpreted as probability density functions. MCMC provides a simple means to marginalise these distributions and to evaluate probability regions.

The setup and procedure of our MCMC analysis closely follows [25], and we refer the reader to this paper for technical details (see also [26-29]). Here we just explain the constraints and priors used in our analysis. We apply the limits from direct SUSY [30] and Higgs $[19,31]$ searches at LEP. The computation of $m_{h}$ suffers from a theoretical uncertainty which has been estimated to amount to up to $2-3 \mathrm{GeV}$ [20]. This theoretical error is most likely non-Gaussian and can give an underestimation as well an overestimation of $m_{h}$. We therefore use the direct experimental search limit for a SM-like Higgs of $m_{h}>114.4 \mathrm{GeV}$ at 


\begin{tabular}{|l|c|l|l|}
\hline Observable & Limit & Likelihood function & Ref. \\
\hline$m_{h}$ & $>114.4$ & $L_{1}(x, 114.5,-0.6)$ & {$[31]$} \\
\hline$m_{t}$ & $173.1 \pm 1.3$ & $L_{2}(x, 173.1,1.3)$ & {$[32]$} \\
\hline$m_{W}$ & $80.398 \pm 0.025$ & $L_{2}(x, 80.398,0.025)$ & {$[33]$} \\
\hline $\mathrm{BR}(b \rightarrow s \gamma)$ & $(3.52 \pm 0.34) \times 10^{-4}$ & $L_{2}\left(x, 3.52 \times 10^{-4}, 0.34 \times 10^{-4}\right)$ & {$[34,35]$} \\
\hline $\mathrm{BR}\left(B_{s} \rightarrow \mu^{+} \mu^{-}\right)$ & $\leq 5.8 \times 10^{-8}$ & $L_{1}\left(x, 5.8 \times 10^{-8}, 5.8 \times 10^{-10}\right)$ & {$[36]$} \\
\hline $\mathrm{R}\left(B_{u} \rightarrow \tau \nu_{\tau}\right)$ & $1.11 \pm 0.52$ & $L_{2}(x, 1.11,0.52)$ & {$[34]$} \\
\hline$\Delta a_{\mu}^{\text {SUSY }}$ & $\leq 4.48 \times 10^{-9}$ & $L_{1}\left(x, 4.48 \times 10^{-9}, 4.5 \times 10^{-11}\right)$ & {$[37]$} \\
\hline$\Omega h^{2}$ & $0.1131 \pm 0.0034$ & $L_{2}(x, 0.113,0.011)$ & {$[38]$} \\
\hline SUSY mass limits & LEP limits & 1 or $10^{-9}$ & {$[30]$} \\
\hline
\end{tabular}

Table 1. Observables used in the likelihood calculation. $L_{1}$ and $L_{2}$ are defined in eq. (4.1).

95\% C.L. without further modification. One should however bear in mind that the favoured regions of parameter space may in fact be somewhat larger (where the Higgs mass is underestimated by the calculation) or smaller (where it is overestimated) than the ones we find.

Regarding the anomalous magnetic moment of the muon, we limit our scans to $\mu>0$, which gives a positive SUSY contribution, but do not require that SUSY explains the discrepancy between the measurement and SM prediction; instead we only apply an upper limit on $\Delta a_{\mu}^{\mathrm{SUSY}}$.

The complete set of constraints applied is given in table 1. For observables on which there is merely an experimental upper or lower bound available, we use a Fermi likelihood function $L_{1}$. For quantities which have been measured, we use a Gaussian likelihood function $L_{2}$. The total likelihood of a parameter point is the product of all individual likelihoods, $L=\prod_{n}\left(L_{i}\right)_{n}$. In the notation of table 1, we have

$$
L_{1}\left(x, x_{0}, d x\right)=\frac{1}{1+\exp \left[\left(x-x_{0}\right) / d x\right]}, \quad L_{2}\left(x, x_{0}, d x\right)=\exp \left[-\frac{\left(x-x_{0}\right)^{2}}{2 d x^{2}}\right]
$$

The neutralino relic density, the B-decay branching ratios, $\Delta a_{\mu}^{\mathrm{SUSY}}$, and the SUSY mass limits are evaluated with micrOMEGAs $[39,40] .{ }^{4}$

We choose to work with two different prior probability distributions. Our first prior is flat in the GUT-scale soft parameters and in $\tan \beta$. That is, within a certain fixed range, any value for a given parameter is treated as equally probable. As a second prior, for comparison, we use a "naturalness prior" [26]: Since a prior choice ultimately reflects theoretical prejudice as to what parameter choices should be more or less likely, we find it appropriate to use a prior which disfavours the more fine-tuned parameter points. The main source for fine-tuning in the MSSM is caused by the sensitivity of the electroweak

\footnotetext{
${ }^{4}$ In the likelihood function for $\Omega h^{2}$, we use the 2008 central value of [38] with a Gaussian width of about $10 \%$. This is to approximately account for uncertainties from the cosmological model, from the data sets used, and from the SUSY spectrum calculation. It is consistent with the most recent determination of $\Omega h^{2}$ from seven-year WMAP data, published in early 2010 [41, 42].
} 
scale to parameter variations. We therefore use a fine-tuning measure $c$ defined as [43]

$$
c=\max _{i}\left|\frac{\partial \ln m_{Z}}{\partial \ln a_{i}}\right| .
$$

Here $\left\{a_{i}\right\}$ includes all GUT-scale soft masses and trilinear soft terms, as well as $\mu$. With the naturalness prior, every parameter point is then weighted with a measure $1 / c$, thus penalizing the more fine-tuned ones.

Before presenting the results, let us comment on the viable dark matter regions. In general in the MSSM there are only a few mechanisms that provide the correct amount of neutralino annihilation consistent with cosmological observations (see e.g. [44] for a review). In the DHMM case we consider here, we expect:

A) Coannihilation with sleptons. This requires small neutralino-slepton mass differences of roughly $10-1 \mathrm{GeV}$ for $m_{\tilde{\chi}_{1}^{0}} \sim 100-500 \mathrm{GeV}$; for heavier LSPs, coannihilation with sleptons alone is not efficient enough. Another possibility is coannihilation with light $\tilde{t}_{1}$ or $\tilde{b}_{1}$, which is efficient for larger mass differences, or larger LSP masses.

B) Annihilation through $s$-channel pseudoscalar Higgs exchange. Here the key quantities are the distance from the $A$ pole, $m_{A}-2 m_{\tilde{\chi}_{1}^{0}}$, and the width of the $A$ resonance. The process is efficient for a bino LSP, although some higgsino admixture is necessary to provide the $\tilde{\chi}_{1}^{0} \tilde{\chi}_{1}^{0} A$ coupling.

C) Annihilation of a mixed bino-higgsino LSP through $t$-channel chargino and neutralino exchange, and through $s$-channel $Z$ exchange. This requires a sizable LSP higgsino fraction $f_{H} \gtrsim 0.25 \%$. Heavier LSPs need a larger higgsino fraction, so that eventually coannihilation with other neutralinos and charginos also becomes important. Besides, if $2 m_{\tilde{\chi}_{1}^{0}} \sim m_{A}, s$-channel $A$ exchange also contributes in this region.

Finally note that throughout this work the squark and slepton mass matrices and $A$ terms are assumed to be diagonal. The issue of flavour-changing neutral currents due to non-diagonal terms arising in particular in the warped case [45-47] is left for a separate work [48].

\subsection{Results for universal soft terms}

Here the model parameters to scan over are universal gaugino and sfermion mass parameters $M_{1 / 2}$ and $m_{0}$, a universal trilinear coupling $A_{0}$, and $\tan \beta$. In addition there are the two discrete parameters $\operatorname{sign}(\mu)$ and $\epsilon_{H}$. We choose $\mu>0$ as favoured by $\operatorname{BR}(b \rightarrow s \gamma)$ and run ten chains with $10^{6}$ iterations each, for both $\epsilon_{H}=+1$ and -1 , allowing $M_{1 / 2}$ to vary from 0 to $2 \mathrm{TeV}, m_{0}$ from 0 to $5 \mathrm{TeV}, A_{0}$ within $\pm 10 \mathrm{TeV}$ and $\tan \beta$ from 2 to 60 .

Figure 7 shows the marginalized 1D posterior probability distributions of the input parameters comparing flat (in black) to natural (in red) prior. The case of $\epsilon_{H}=-1$ is shown in figure 8. As can be seen, in both cases the naturalness prior results in a pull towards smaller masses and smaller $\tan \beta$. The general features, which are detailed below, however remain the same. 

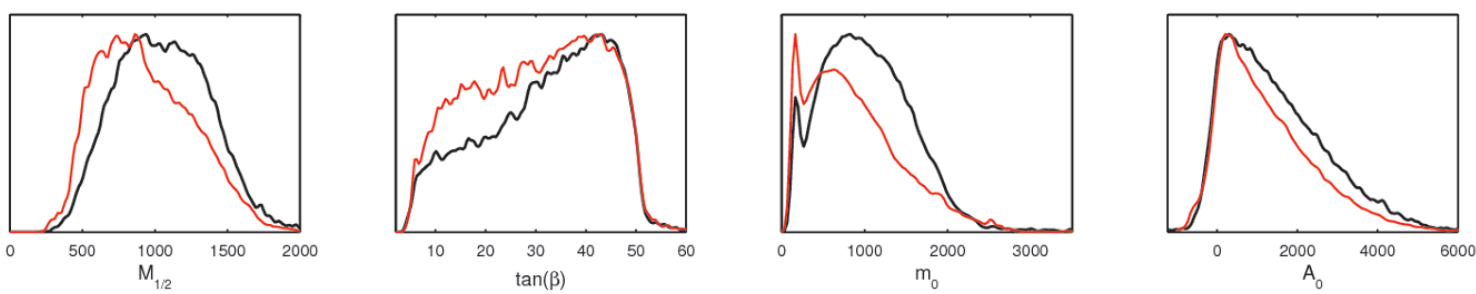

Figure 7. Marginalized 1D posterior probability distributions of the input parameters for universal soft terms and $\epsilon_{H}=+1$. Black lines are for flat prior, red lines for natural prior. Dimensionful quantities are in $\mathrm{GeV}$.
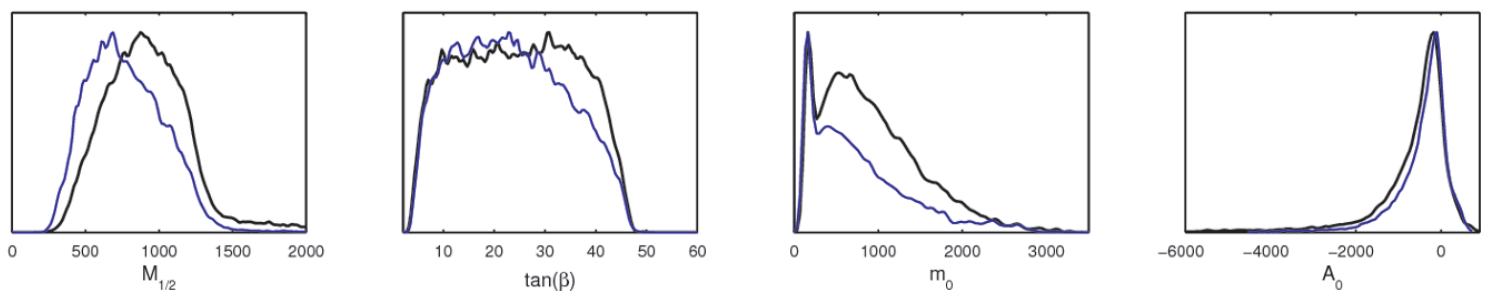

Figure 8. Same as figure 7 but for $\epsilon_{H}=-1$; black (blue) lines are for flat (natural) prior.

$M_{1 / 2}$ is bounded from below by the Higgs and SUSY mass limits, and from above by the requirement of sufficient neutralino annihilation. The processes that bring the neutralino relic density within the desired range are A) or B) from above: coannihilation with sleptons $\left(\tilde{e}_{R}, \tilde{\mu}_{R}\right.$, or $\left.\tilde{\tau}_{1}\right)$ or annihilation through the Higgs funnel. On the other hand, we do not find any region where the LSP higgsino fraction is large enough to render processes C) efficient. Coannihilation with stops or sbottoms is also absent. For $\epsilon_{H}=+1$ it becomes difficult to achieve small enough $\left|m_{A}-2 m_{\tilde{\chi}_{1}^{0}}\right|$ and $m_{\tilde{l}}-m_{\tilde{\chi}_{1}^{0}}$ when $m_{\tilde{\chi}_{1}^{0}} \gtrsim 750-800 \mathrm{GeV}$. For $\epsilon_{H}=-1$ this is the case when $m_{\tilde{\chi}_{1}^{0}} \gtrsim 600 \mathrm{GeV}$.

The relic density constraint also prefers higher $\tan \beta$, for which the Higgs funnel is more efficient. This is the reason for the preference of high $\tan \beta$ in the distribution for $\epsilon_{H}=+1$ and flat prior (which is still softened by the natural prior). High values around $\tan \beta \sim 50$ are constrained by $\operatorname{BR}(b \rightarrow s \gamma)$ becoming too low. For $\epsilon_{H}=-1$, the $\tan \beta$ distribution is more flat because the $\mathrm{BR}(b \rightarrow s \gamma)$ constraint becomes effective earlier as $\tan \beta$ grows. These correlations are illustrated in figure 9 .

Regarding the $m_{0}$ probability distribution, the peak at low $m_{0}$ is where coannihilation with sleptons takes place. Slepton coannihilation, and with it the low $m_{0}$ peak, becomes more relevant when using the natural prior because of its preference for smaller $\tan \beta$ for which the Higgs funnel is less efficient. Overall, however, the annihilation through the pseudoscalar resonance is by far the dominant mechanism: for $\epsilon_{H}=+1$ and flat (natural) prior, $88 \%$ (83\%) of the points exhibit predominantly annihilation into $b \bar{b}$, while $10 \%(15 \%)$ predominantly show coannihilation with sleptons. For $\epsilon_{H}=-1$, the $A$ resonance is more difficult to hit, partly because $\tan \beta$ is smaller, so that for flat (natural) prior $22 \%(26 \%)$ of the points predominantly show slepton coannihilation. 

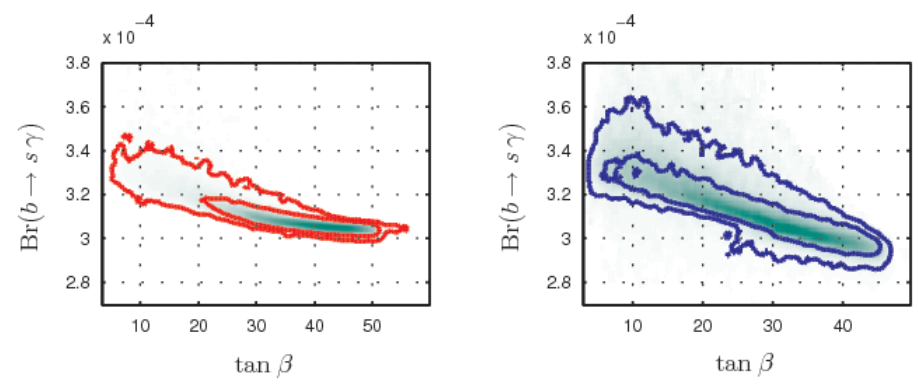

Figure 9. Contours of $68 \%$ and $95 \%$ probability in the $\mathrm{BR}(b \rightarrow s \gamma)$ versus $\tan \beta$ plane, on the left for $\epsilon_{H}=+1$, on the right for $\epsilon_{H}=-1$. The green shading maps the average likelihood per bin, normalized to the maximum likelihood.
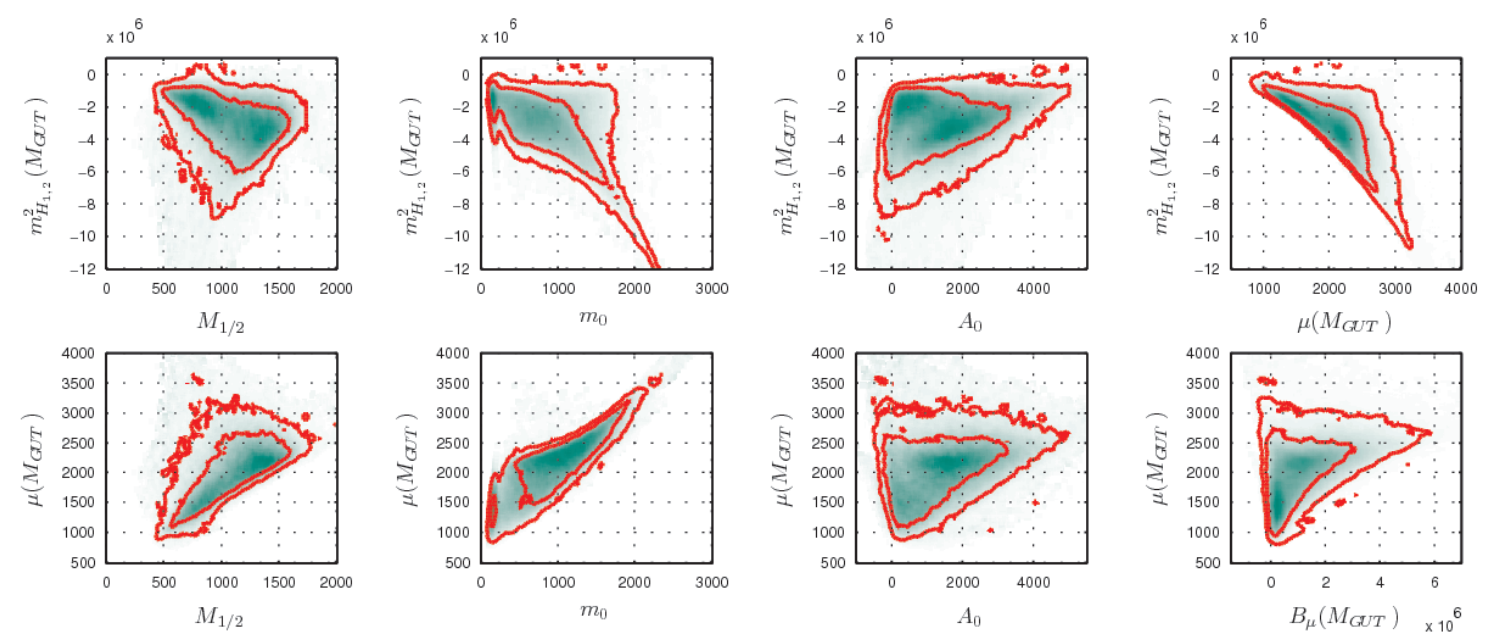

Figure 10. Contours of $68 \%$ and $95 \%$ probability showing correlations between $m_{H_{1,2}}^{2}, \mu, B_{\mu}$ and the input parameters for universal soft terms, $\epsilon_{H}=+1$ and flat prior.

As opposed to the CMSSM there is no "focus point" behaviour in this scenario: The $m_{0}$ distribution shows a clear preference for lower values $\lesssim 2 \mathrm{TeV}$, and $m_{0}$ is significantly correlated with $\mu$. In fact the CMSSM focus point hinges on having a single parameter which governs the scalar soft masses for both Higgs and matter fields. This is clearly not the case in DHMM models.

Finally, the $A_{0}$ distribution confirms our discussion of the sign correlations in section 3.

An important issue in our considerations are the values of $m_{H_{1,2}}^{2}, \mu$ and $B_{\mu}$ at the GUT scale resulting from the DHMM condition. In figures 10 and 11 we therefore show 2D posterior probability distributions for these parameters. ${ }^{5}$ The tight correlation between $m_{H_{1,2}}^{2}$ and $\mu$ is clearly visible. Moreover, as can be seen, for both $\epsilon_{H}= \pm 1$ small $\left|m_{H_{1,2}}^{2}\right|$ and $|\mu|$ prefers small values of $M_{1 / 2}, m_{0}$ and $\epsilon_{H} A_{0}$.

Regarding consequences for experiments, figures 12 and 13 show 1D posterior probability distributions for SUSY and Higgs masses. Also shown are the distributions for the

\footnotetext{
${ }^{5}$ To limit the proliferation of figures we only show $2 \mathrm{D}$ distributions for flat prior; those for natural prior look very similar.
} 

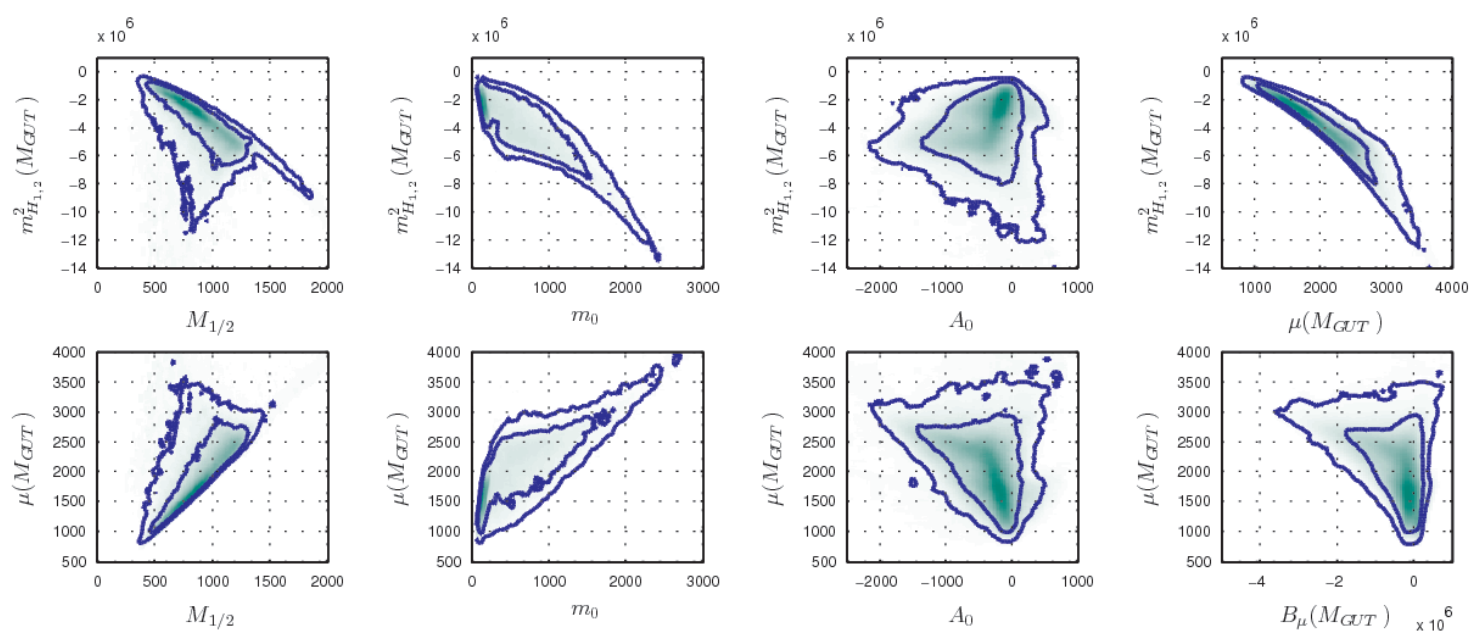

Figure 11. Same as figure 10 but for $\epsilon_{H}=-1$.

LSP higgsino fraction $f_{H}$ and the cross section for spin-independent direct detection $\sigma_{\chi p}^{\mathrm{SI}}$. The pull of the natural prior towards lighter masses and in particular towards smaller $\mu$ is again evident. We also note that most of the parameter space lies within reach of the LHC at $14 \mathrm{TeV}$ centre-of-mass energy. In fact, for $\epsilon_{H}=+1(-1)$ and flat prior, $82 \%(97 \%)$ of the points have gluino and squark masses below $3 \mathrm{TeV}$. Moreover, $55 \%(58 \%)$ of these points have sleptons that are lighter than the $\tilde{\chi}_{2}^{0}$, so that a same-flavour opposite-sign dilepton signal from $\tilde{\chi}_{2}^{0} \rightarrow \tilde{\ell}^{ \pm} \ell^{\mp} \rightarrow \ell^{ \pm} \ell^{\mp} \tilde{\chi}_{1}^{0}$ may be visible in SUSY cascade decays (if decays into sleptons are absent or kinematically suppressed, then $\tilde{\chi}_{2}^{0} \rightarrow h \tilde{\chi}_{1}^{0}$ is the most important decay mode of $\tilde{\chi}_{2}^{0}$ ). For naturalness prior, $88 \%$ (99\%) of the $\epsilon_{H}=-1$ points have gluino and squark masses below $3 \mathrm{TeV}$, with $64 \%$ of these featuring $m_{\tilde{e}, \tilde{\tau}}<m_{\tilde{\chi}_{2}^{0}}$.

If the $\tilde{\chi}_{2}^{0}$ decay into sleptons is open, $\tilde{\chi}_{2}^{0} \rightarrow \tilde{e}^{ \pm} e^{\mp}, \tilde{\mu}^{ \pm} \mu^{\mp}$ has up to about $40 \%$ branching ratio. It is however important to keep in mind that owing to the universality assumption, the typical mass ordering is $m_{\tilde{\tau}_{1}}<m_{\tilde{e}_{R}}<m_{\tilde{e}_{L}}$. Therefore $\tilde{\chi}_{2}^{0} \rightarrow \tilde{\tau}_{1}^{ \pm} \tau^{\mp}$ decays are often dominant.

Concerning direct dark matter detection, we note that because the LSP is always almost a pure bino, the neutralino scattering cross section on proton is typically of the order of $10^{-11}-10^{-10} \mathrm{pb}$ and hence beyond the reach of current experiments.

Finally, we observe that even with the natural prior the fine-tuning tends to be very large, of the level of per-mil, and points with $c<100$, corresponding to less than $1 \%$ fine-tuning are difficult to obtain. Correlations of the finetuning measure $c$ are illustrated in figure 14 for natural prior. The lowest fine-tuning occurs for small $M_{1 / 2}$, medium $\tan \beta \sim 20-30, A_{0} \sim 0$ and $m_{0} \sim 1 \mathrm{TeV}$, with $\mu$ being around $1.5-2 \mathrm{TeV}$.

\subsection{Results for vanishing $1 \mathrm{st} / 2 \mathrm{nd}$ generation soft terms}

Let us now turn to the pattern of soft terms obtained from models such as the gauge-Higgs unification (GHU) model of section 2.1. Here the first- and second-generation matter fields were localized on a brane and SUSY breaking was mediated by the radion, leading to 

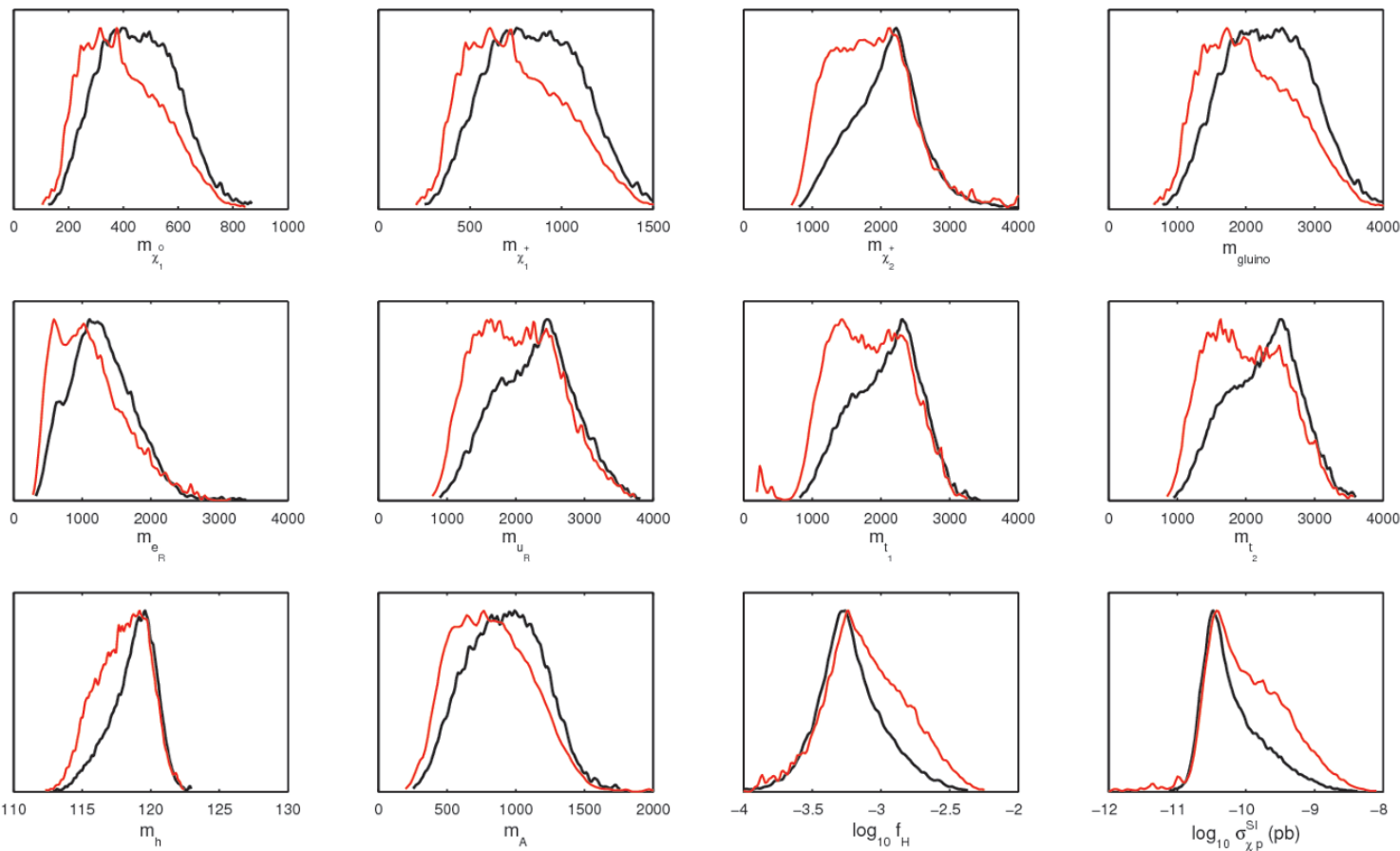

Figure 12. Posterior probability distributions of the most relevant masses for universal soft terms and $\epsilon_{H}=+1$. The bottom-right plots show the LSP higgsino fraction, $f_{H}:=\left|N_{13}\right|^{2}+\left|N_{14}\right|^{2}$, and the spin-independent scattering cross section on protons. As above, black lines are for flat and red lines for natural prior.

vanishing 1st/2nd generation and non-universal 3rd generation soft terms. We will call this "GHU-like boundary conditions" in the following. The free parameters in this case are $M_{1 / 2}, \tan \beta$, and the third-generation soft terms $m_{Q_{3}}, m_{U_{3}}, m_{D_{3}}, A_{t}, A_{b}, m_{L_{3}}, m_{E_{3}}, A_{\tau}$. We allow $M_{1 / 2}$ to vary from 0 to $2 \mathrm{TeV}, \tan \beta$ from 2 to $60, m_{Q_{3}, U_{3}, D_{3}}^{2}$ within $\pm 25 \mathrm{TeV}^{2}$, $m_{L_{3}, E_{3}}^{2}$ from 0 to $4 \mathrm{TeV}^{2}$, and $A_{t, b, \tau}$ within $\pm 10 \mathrm{TeV}$.

The marginalized 1D posterior probability distributions of the input parameters are displayed in figure 15 for $\epsilon_{H}=+1$ and in figure 16 for $\epsilon_{H}=-1$. Analogously, figures 17 and 18 show the probability distributions of masses, $\mu$ parameter, LSP higgsino fraction, and the spin-independent LSP scattering cross section on protons.

Two important differences to the case of universal soft terms are that $M_{1 / 2}$ can now go to much higher values, and that $\tan \beta$ peaks around 10 . The reason is on the one hand that due to the no-scale boundary conditions for the 1st/2nd generation, coannihilation with selectrons and smuons becomes more likely; this is mainly relevant for $m_{\tilde{\chi}_{1}^{0}} \lesssim 500 \mathrm{GeV}$. Accordingly, there are distinct peaks at $m_{\tilde{\chi}_{1}^{0}} \approx 400 \mathrm{GeV}$ in figures 17 and 18 , corresponding to the peaks at $M_{1 / 2} \approx 900 \mathrm{GeV}$ in figures 15 and 16 . On the other hand, due to the non-universal 3rd generation we can obtain smaller values of $\mu$, and hence processes $\mathrm{C}$ ) become important. This is mainly relevant for heavy $\tilde{\chi}_{1}^{0}$ and leads to the peak at large $M_{1 / 2}$ for $\epsilon_{H}=+1$. For $\epsilon_{H}=-1, \mu$ tends to be larger (i.e. $f_{H}$ tends to be smaller) and consequently the high $M_{1 / 2}$ region is less favoured. Besides, for both $\epsilon_{H}= \pm 1$, we find 

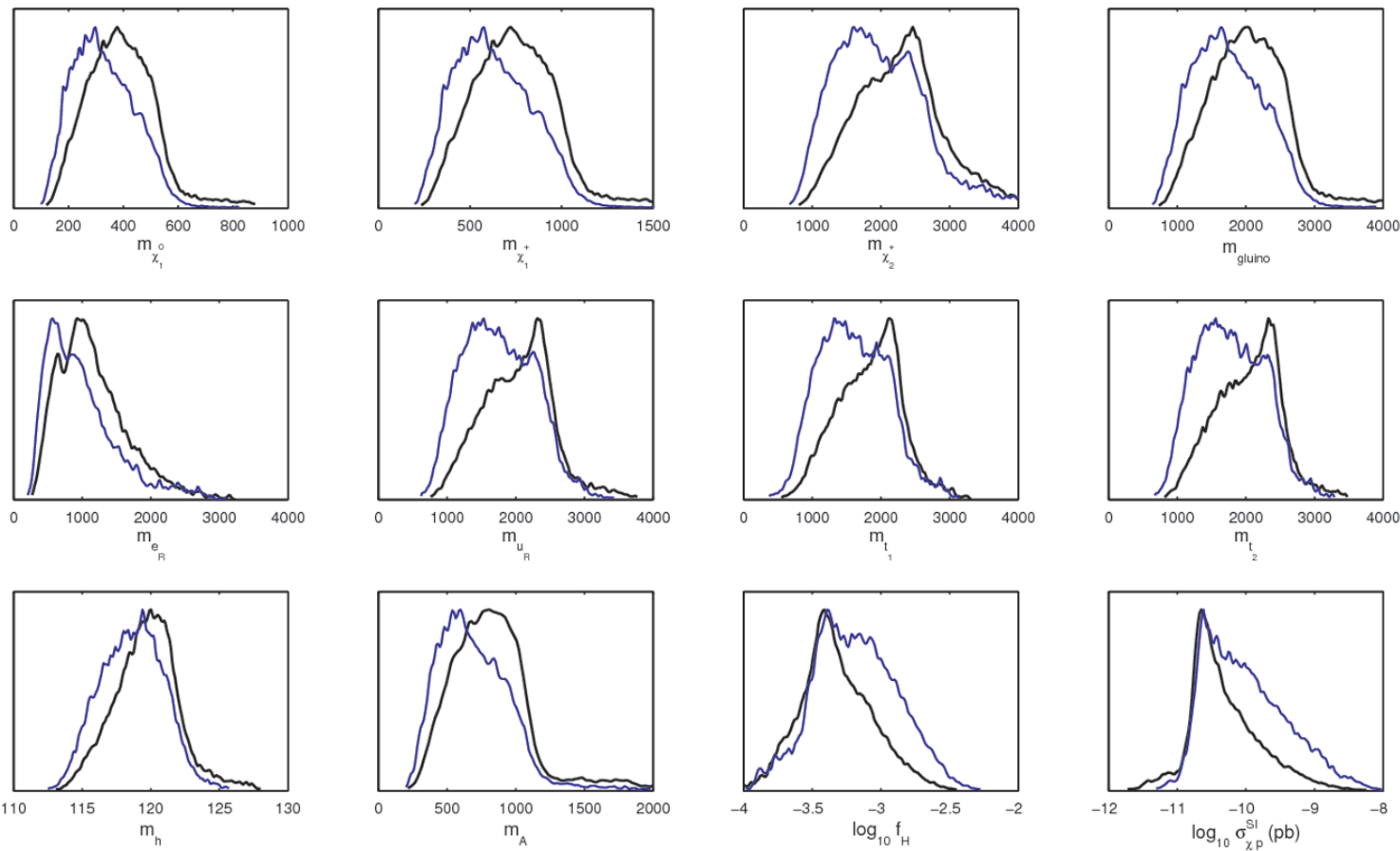

Figure 13. Same as figure 12 but for $\epsilon_{H}=-1$; black (blue) lines are for flat (natural) prior.
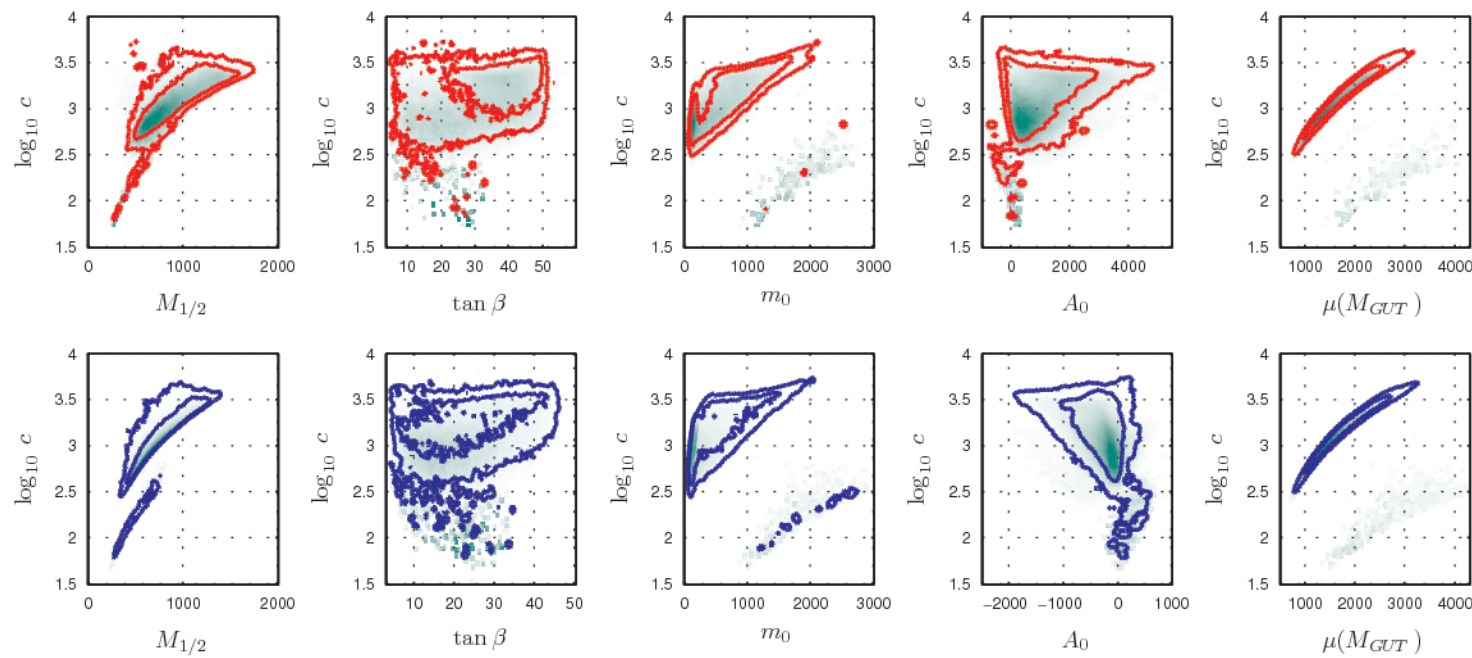

Figure 14. 2D posterior probability distributions of the fine-tuning measure $c$ for natural prior. The contours enclose regions of $68 \%$ and $95 \%$ probability. The top row (red contours) is for $\epsilon_{H}=+1$, the bottom row (blue contours) for $\epsilon_{H}=-1$.

some coannihilation with $\tilde{b}_{1}$ and/or $\tilde{t}_{1}$, though this is diminished by the naturalness prior. (For $\epsilon_{H}=-1$, this leads to the peak at large negative $m_{D_{3}}$, which gives light $\tilde{b}_{1} \sim \tilde{b}_{R}$, c.f. figure 18. Coannihilation with $\tilde{t}_{1}$ is less frequent, in particular for $\epsilon_{H}=+1$, as the Higgs mass bound pushes the stop masses up.)

Concerning collider phenomenology, we first observe that, because of the vanishing 1st/2nd generation soft terms, the $\tilde{\chi}_{2}^{0} \rightarrow \tilde{\ell}^{ \pm} \ell^{\mp} \rightarrow \ell^{ \pm} \ell^{\mp} \tilde{\chi}_{1}^{0}$ decay, with $\ell=e$ or $\mu$, is almost 

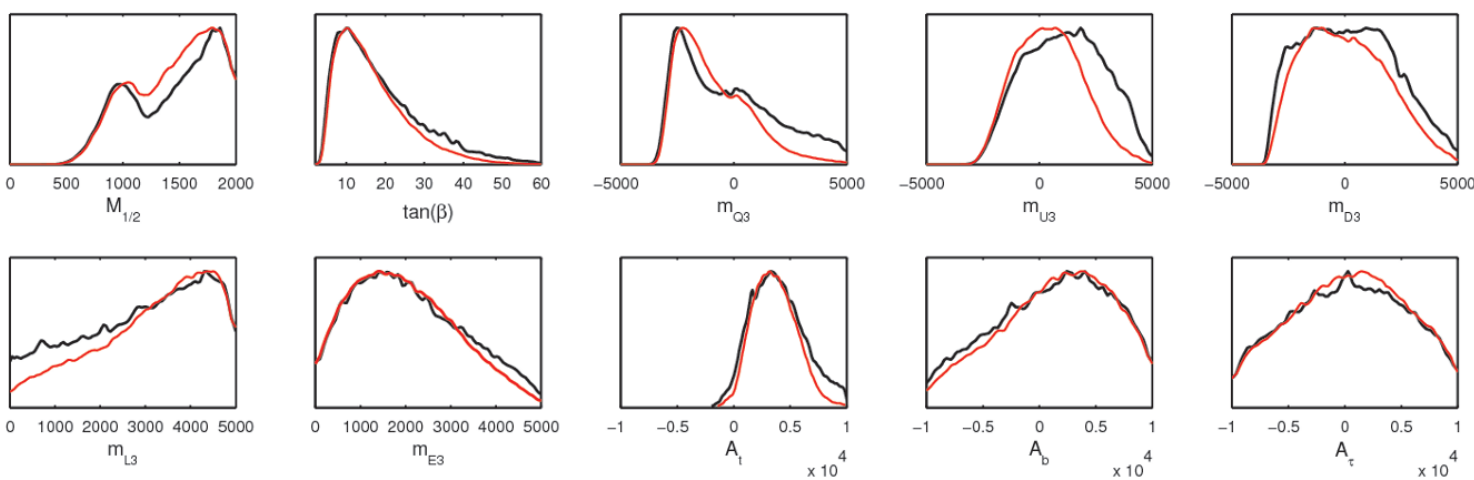

Figure 15. Marginalized 1D posterior probability distributions of the input parameters for GHUlike boundary conditions and $\epsilon_{H}=+1$. Black lines are for flat prior, red lines for natural prior. Dimensionful quantities are in $\mathrm{GeV}$.
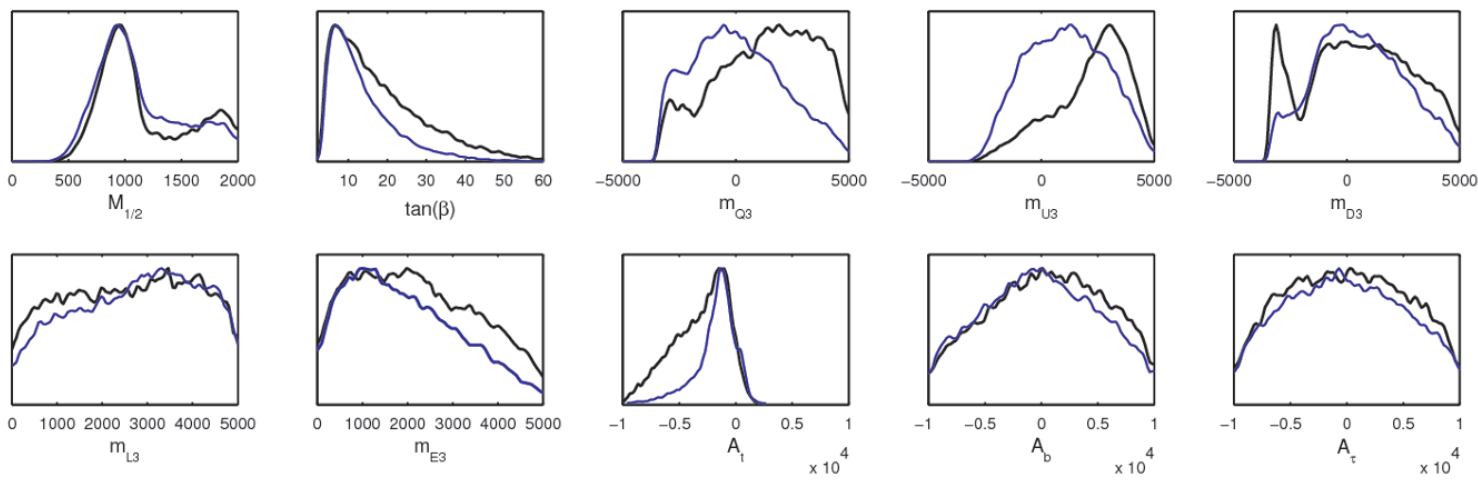

Figure 16. Same as figure 15 but for $\epsilon_{H}=-1$; black (blue) lines are for flat (natural) prior.

always present. Staus are heavier and hence much less important for the $\tilde{\chi}_{2}^{0}$ decays. Second, for $\epsilon_{H}=-1$, squark and gluino masses peak around $2 \mathrm{TeV}$, which means that the LHC at $14 \mathrm{TeV}$ centre-of-mass energy has again a very good discovery potential over most of the parameter space. More precisely, $88 \%$ of the $\epsilon_{H}=-1$ points have $m_{\tilde{q}, \tilde{g}} \leq 3 \mathrm{TeV}$. For $\epsilon_{H}=+1$, on the other hand, we find that a considerable fraction of the parameter space lies beyond the reach of the LHC. In this region the $\tilde{\chi}_{1}^{0}$ is heavy and is very likely to have a large higgsino fraction (since we require $\Omega h^{2} \sim 0.1$ ). In turn this leads to a large cross section for direct dark matter detection of up to around $10^{-7} \mathrm{pb}$, see the bottom right plot in figure 17: Interestingly, this is just at the edge of current CDMS-II exclusion limit [49] for heavy masses. ${ }^{6}$ The $2 \mathrm{D}$ probability distributions in the plane $\sigma_{\chi p}^{\mathrm{SI}}$ versus $m_{\tilde{\chi}_{1}^{0}}$ are shown in figure 19 for the natural prior. It is very gratifying that these models can be experimentally tested with complimentary methods, by both LHC and direct dark matter searches.

For completeness, figures 20 to 23 show various parameter correlations in $2 \mathrm{D}$. It is interesting to see that $m_{H_{1,2}}^{2}\left(M_{\mathrm{GUT}}\right)=0$ is easily obtained for $\epsilon_{H}=+1$, but does not occur for $\epsilon_{H}=-1$. Moreover, the sign correlation between $\epsilon_{H}, B_{\mu}$ and $A_{t}$ discussed in section 3 is evident.

\footnotetext{
${ }^{6}$ While we have not used constraints from direct dark matter searches in the MCMC, a posteriori it
} 

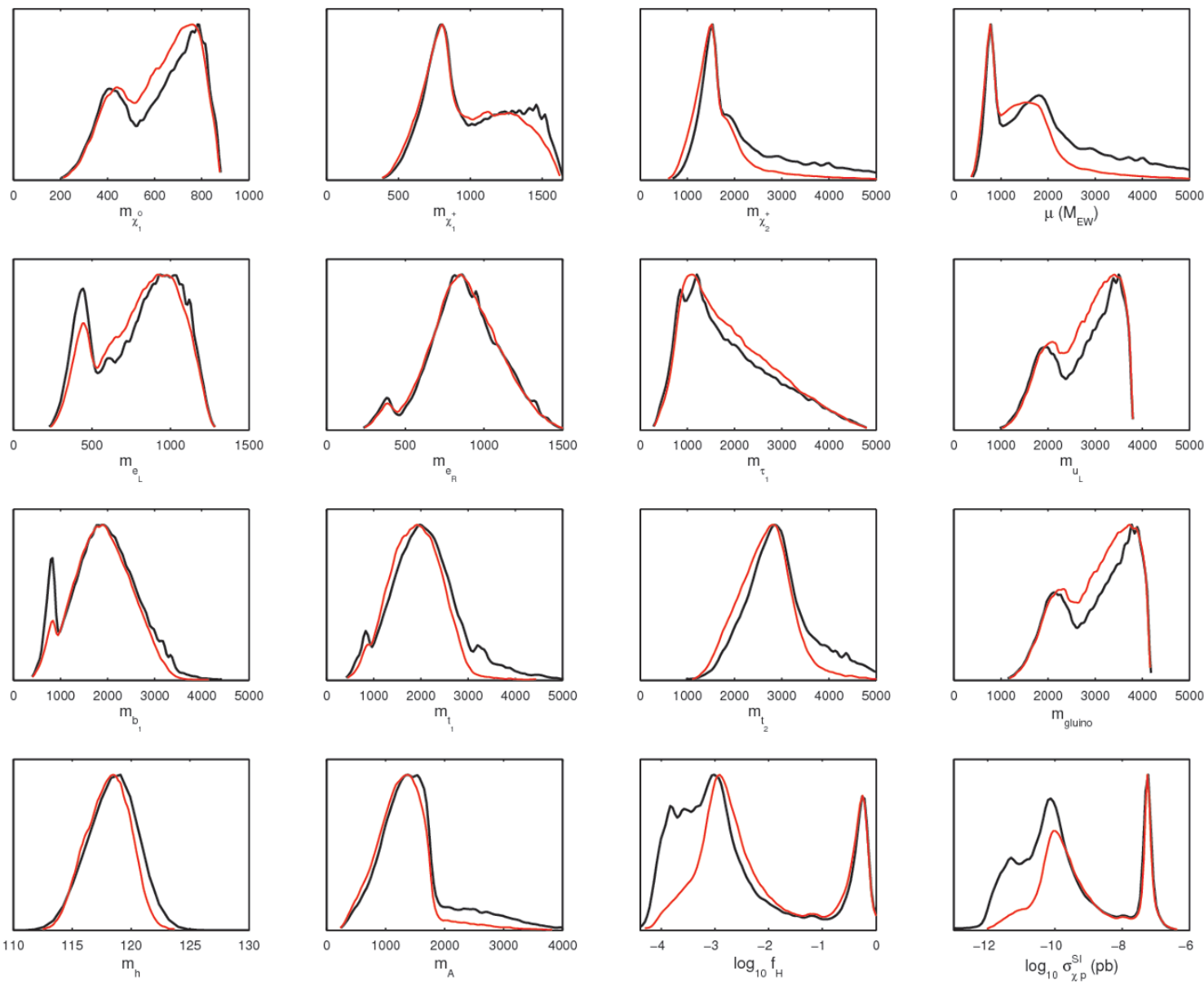

Figure 17. Posterior probability distributions of the most relevant masses, $\mu\left(M_{\mathrm{EW}}\right)$, LSP higgsino fraction, and the spin-independent LSP scattering cross section on protons for GHU-like boundary conditions with $\epsilon_{H}=+1$. As above, black lines are for flat and red lines for natural prior.

\section{Conclusions}

Among the many possible embeddings of the MSSM into a grand-unified theory, there are some interesting classes of models which predict a degenerate GUT-scale Higgs mass matrix. We have investigated the origin of this prediction in some example high-scale models, as well as its consequences for low-scale mass spectra and phenomenology.

With the additional assumption of universal GUT-scale gaugino masses (which is valid in most simple GUT scenarios) the low-energy spectrum still depends sensitively on the sfermion soft terms. Different high-scale models will give rise to various patterns of sfermion masses and trilinear terms. We have chosen to investigate two representative cases in detail: first, universal sfermion soft terms, and second, vanishing soft terms for the first two generations but non-vanishing and non-universal ones for the third. Both these cases are well motivated from the GUT model building point of view.

turns out that only about $1 \%$ of the points with higgsino LSP violate the current limits. 

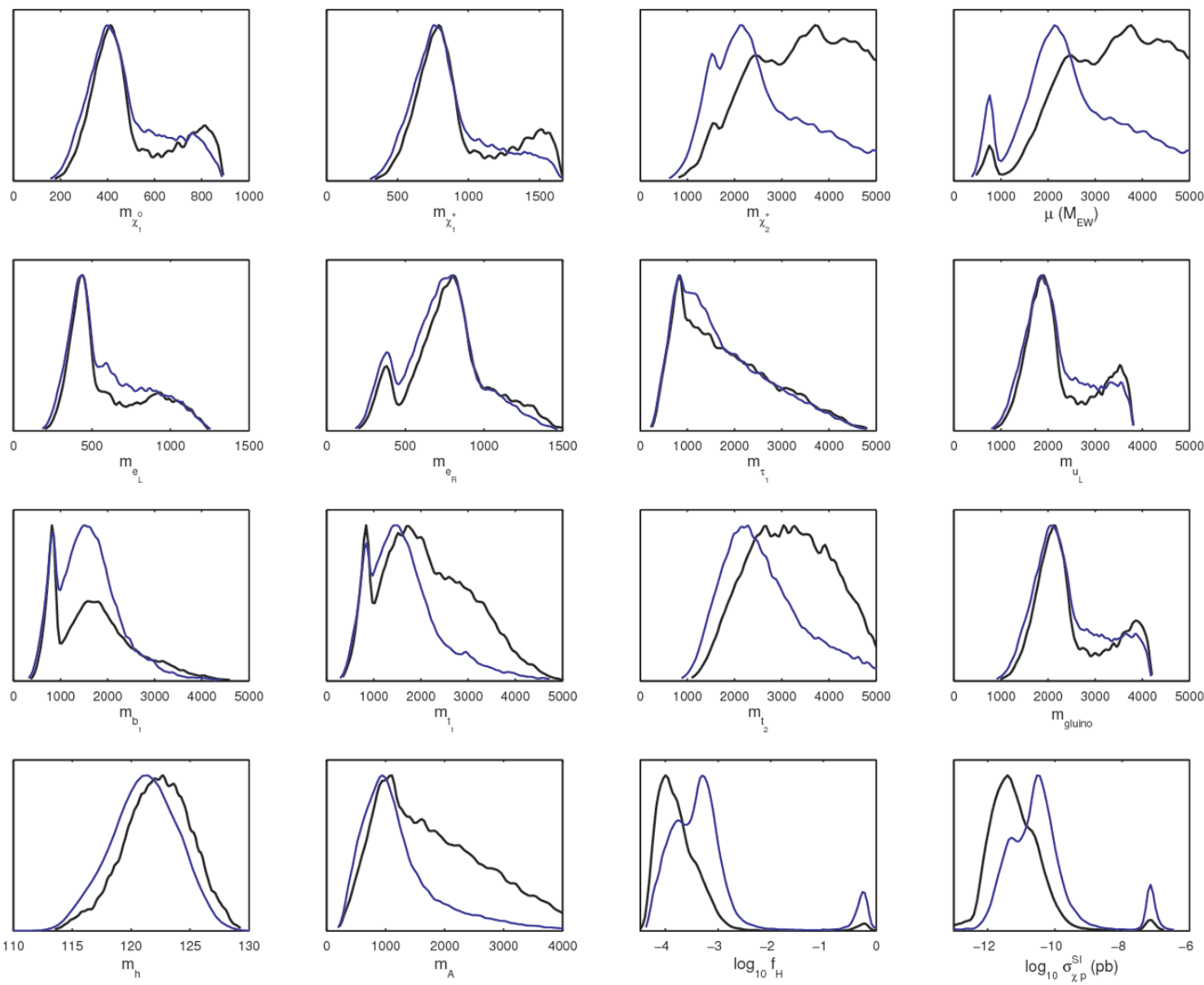

Figure 18. Same as figure 17 but for $\epsilon_{H}=-1$; black (blue) lines are for flat (natural) prior.
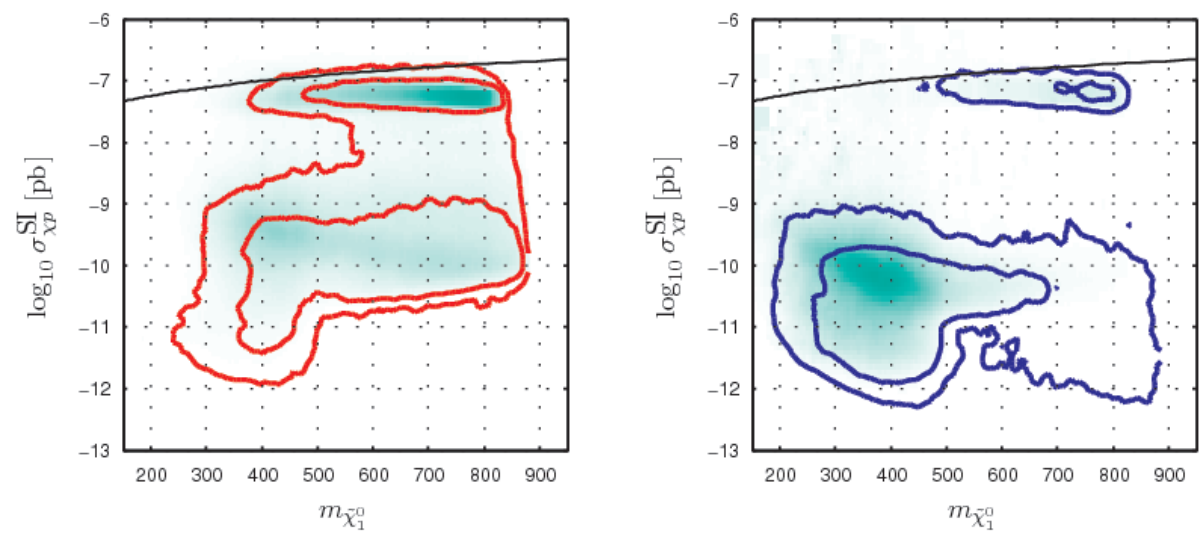

Figure 19. Probability distributions in the plane $\sigma_{\chi p}^{\mathrm{SI}}$ versus $m_{\tilde{\chi}_{1}^{0}}$ for naturalness prior, on the left for $\epsilon_{H}=+1$, on the right for $\epsilon_{H}=-1$. The inner (outer) contours enclose regions of $68 \%(95 \%)$ probability, the green shading maps the average likelihood, and the black lines show the limit from CDMS-II, which is currently providing the strongest bound in this mass range. 

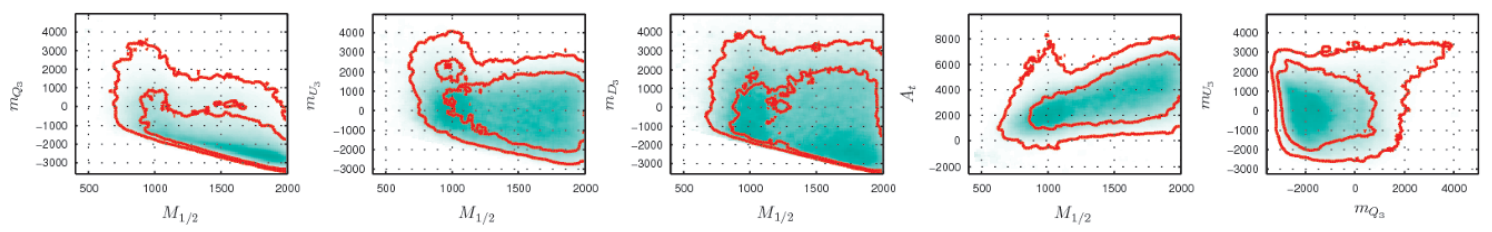

Figure 20. Contours of $68 \%$ and $95 \%$ probability showing correlations between the most relevant input parameters for GHU-like boundary conditions, $\epsilon_{H}=+1$ and naturalness prior.
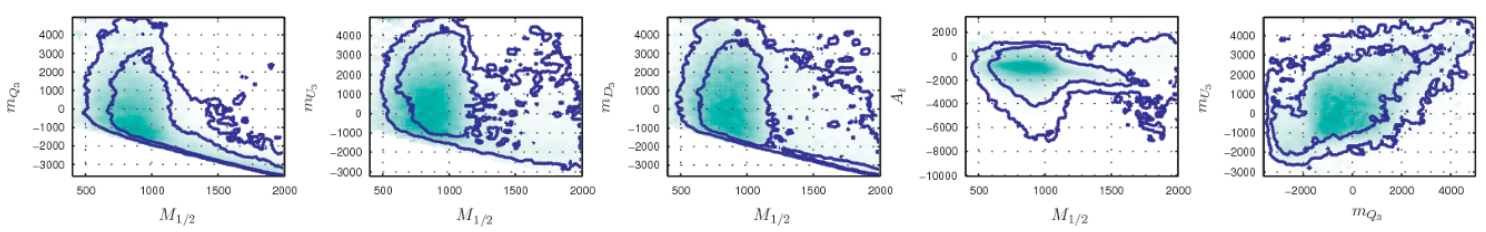

Figure 21. Same as figure 20 but for $\epsilon_{H}=-1$.
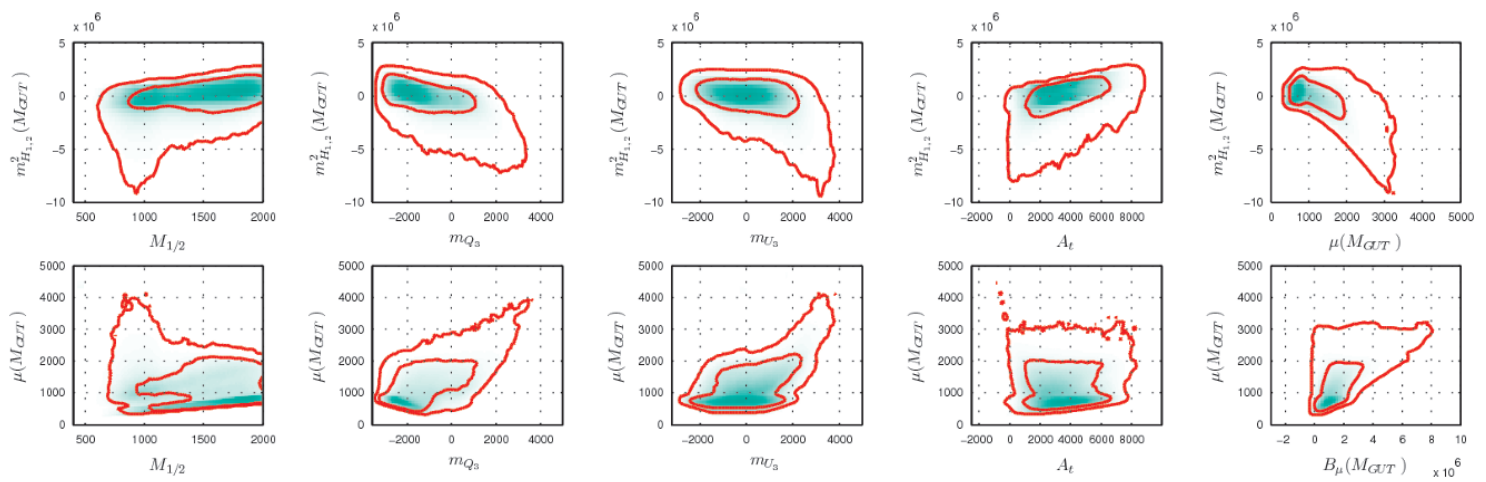

Figure 22. Contours of $68 \%$ and $95 \%$ probability showing correlations between $m_{H_{1,2}}^{2}, \mu, B_{\mu}$ and the most relevant input parameters for GHU-like boundary conditions, $\epsilon_{H}=+1$ and naturalness prior.
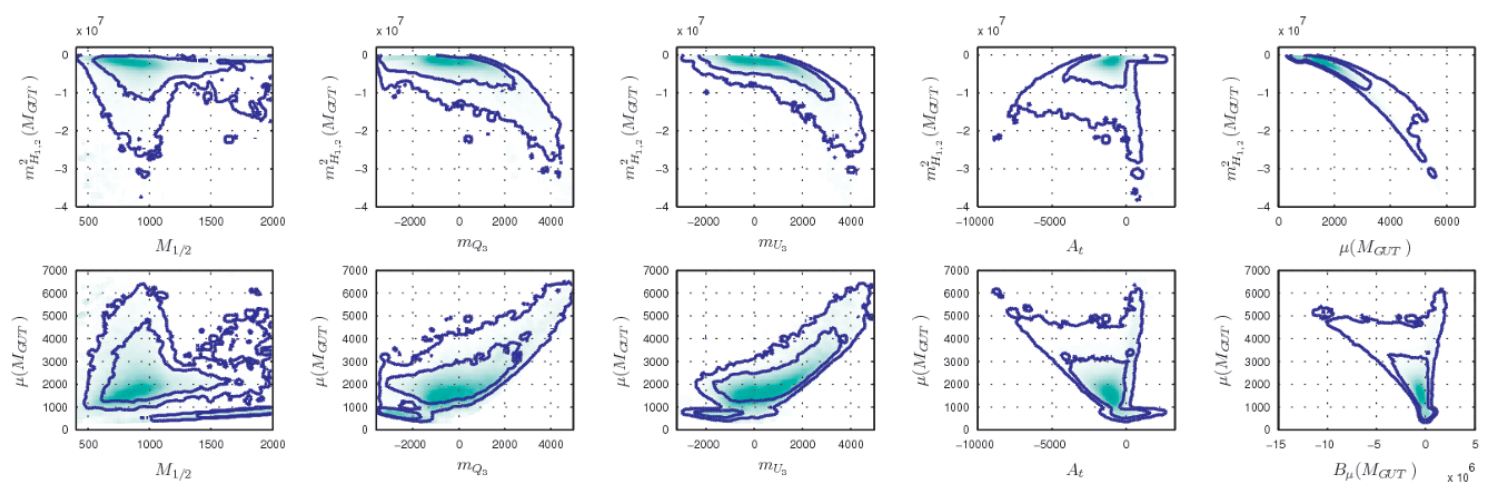

Figure 23. Same as figure 22 but for $\epsilon_{H}=-1$. 
We explained how the remaining independent high-scale parameters are constrained by the requirement of realistic electroweak symmetry breaking and a sufficiently large Higgs mass. We also briefly compared with the related CMSSM and NUHM scenarios. Finally we presented a detailed parameter scan using Markov Chain Monte Carlo methods, highlighting the preferred ranges of parameters as well as correlations between them.

Our analysis shows that models with degenerate Higgs mass matrix can be viable UVcompletions of the MSSM for large ranges of gaugino and sfermion soft terms. They are, however, already strongly constrained by direct Higgs and SUSY searches, flavour physics, and cosmology (as is the MSSM as a whole). In particular, the need to evade the LEP Higgs mass bound leads to preferred sparticle masses in the TeV range. This implies large finetuning in obtaining the correct electroweak scale. Most of the parameter points we found are fine-tuned on the sub-percent level, which of course reflects nothing but the well-known little hierarchy problem of the MSSM. Another stringent constraint arises from the dark matter relic density: In the models we considered, the neutralino relic density is generically larger than the observed value, so rather special parameter values are necessary in order to enhance the neutralino annihilation cross section.

Nevertheless, we find $\Omega h^{2} \simeq 0.1$ over a large part of the parameter space. This is mainly due to Higgs funnel annihilation, a large $\tilde{\chi}_{1}^{0}$ higgsino fraction, or coannihilation with sleptons. In the case of universal sfermion soft terms, the Higgs funnel is clearly the most important process. Here it is worth noting that the shapes of the 1D posterior probability distributions are more or less generated by just demanding correct EWSB, with the other constraints adding little to the shapes. In other words, the EWSB condition already selects the parameters such that most of the low energy observables are of roughly the correct magnitude, with exception of the relic density. It is then mainly the relic density constraint that helps shape the likelihood maps, and this reshaping can be understood in terms of the different (co-)annihilation channel contributions. The global features of the probability distributions are also quite robust against the fine-tuning prior.

Most of the parameter space lies within the reach of $\mathrm{LHC}$ at $14 \mathrm{TeV}$. In the region which is most difficult for the LHC to access, the LSP is higgsino-like and spin-independent direct dark matter detection experiments should soon see a signal. Should the MSSM with degenerate Higgs mass matrix be realized in nature, it will therefore almost certainly be observed within the next few years. This naturally raises the question of model discrimination: Can we look for a piece of experimental evidence pointing more or less uniquely to DHMM models? Unfortunately it seems to us that there is no such "smoking gun" signature for this kind of scenario. LHC may be able to exclude our models, but even if, conversely, an MSSM spectrum compatible with DHMM was found, it would need a future linear collider to accurately measure the sparticle masses and make a bottom-up reconstruction of the GUT-scale structure feasible.

\section{Acknowledgments}

We are indebted to Sezen Sekmen for invaluable help in running the MCMCs. This work is supported by the French ANR project ToolsDMColl, BLAN07-2-194882. The work of 
R.K.S. is supported by the German Ministry of Education and Research (BMBF) under contract 05HT6WWA. F.B. and R.K.S. would like to thank LPSC Grenoble for hospitality and support during various stages of this project.

Open Access. This article is distributed under the terms of the Creative Commons Attribution Noncommercial License which permits any noncommercial use, distribution, and reproduction in any medium, provided the original author(s) and source are credited.

\section{References}

[1] K. Inoue, A. Kakuto and H. Takano, Higgs as (pseudo)Goldstone particles, Prog. Theor. Phys. 75 (1986) 664 [SPIRES].

[2] A.A. Anselm and A.A. Johansen, SUSY GUT with automatic doublet-triplet hierarchy, Phys. Lett. B 200 (1988) 331 [SPIRES].

[3] K.-W. Choi et al., Electroweak symmetry breaking in supersymmetric gauge-Higgs unification models, JHEP 02 (2004) 037 [hep-ph/0312178] [SPIRES].

[4] F. Brümmer, S. Fichet, A. Hebecker and S. Kraml, Phenomenology of supersymmetric gauge-Higgs unification, JHEP 08 (2009) 011 [arXiv:0906.2957] [SPIRES].

[5] G. Burdman and Y. Nomura, Unification of Higgs and gauge fields in five dimensions, Nucl. Phys. B 656 (2003) 3 [hep-ph/0210257] [SPIRES].

[6] A. Hebecker, J. March-Russell and R. Ziegler, Inducing the $\mu$ and the $B_{\mu}$ term by the radion and the 5D Chern-Simons term, JHEP 08 (2009) 064 [arXiv:0801.4101] [SPIRES].

[7] A. Hebecker, $5 D$ super Yang-Mills theory in 4D superspace, superfield brane operators and applications to orbifold GUTs, Nucl. Phys. B 632 (2002) 101 [hep-ph/0112230] [SPIRES].

[8] I. Antoniadis, E. Gava, K.S. Narain and T.R. Taylor, Effective $\mu$ term in superstring theory, Nucl. Phys. B 432 (1994) 187 [hep-th/9405024] [SPIRES].

[9] A. Brignole, L.E. Ibáñez, C. Muñoz and C. Scheich, Some issues in soft SUSY breaking terms from dilaton/moduli sectors, Z. Phys. C 74 (1997) 157 [hep-ph/9508258] [SPIRES].

[10] A. Brignole, L.E. Ibáñez and C. Muñoz, Orbifold-induced $\mu$ term and electroweak symmetry breaking, Phys. Lett. B 387 (1996) 769 [hep-ph/9607405] [SPIRES].

[11] A. Brignole, L.E. Ibáñez and C. Muñoz, Soft supersymmetry-breaking terms from supergravity and superstring models, hep-ph/9707209 [SPIRES].

[12] F. Brümmer, R. Kappl, M. Ratz and K. Schmidt-Hoberg, Approximate R-symmetries and the $\mu$ term, JHEP 04 (2010) 006 [arXiv: 1003.0084] [SPIRES].

[13] Y. Nomura, D. Poland and B. Tweedie, Holographic grand unification, JHEP 12 (2006) 002 [hep-ph/0605014] [SPIRES].

[14] L. Randall and R. Sundrum, Out of this world supersymmetry breaking, Nucl. Phys. B 557 (1999) 79 [hep-th/9810155] [SPIRES].

[15] G.F. Giudice, M.A. Luty, H. Murayama and R. Rattazzi, Gaugino mass without singlets, JHEP 12 (1998) 027 [hep-ph/9810442] [SPIRES].

[16] B.C. Allanach, SOFTSUSY: a C++ program for calculating supersymmetric spectra, Comput. Phys. Commun. 143 (2002) 305 [hep-ph/0104145] [SPIRES]. 
[17] J.R. Ellis, T. Falk, K.A. Olive and Y. Santoso, Exploration of the MSSM with non-universal Higgs masses, Nucl. Phys. B 652 (2003) 259 [hep-ph/0210205] [SPIRES].

[18] H. Baer, A. Mustafayev, S. Profumo, A. Belyaev and X. Tata, Direct, indirect and collider detection of neutralino dark matter in SUSY models with non-universal Higgs masses, JHEP 07 (2005) 065 [hep-ph/0504001] [SPIRES].

[19] LEP Working Group for Higgs boson searches collaboration, R. Barate et al., Search for the standard model Higgs boson at LEP, Phys. Lett. B 565 (2003) 61 [hep-ex/0306033] [SPIRES].

[20] G. Degrassi, S. Heinemeyer, W. Hollik, P. Slavich and G. Weiglein, Towards high-precision predictions for the MSSM Higgs sector, Eur. Phys. J. C 28 (2003) 133 [hep-ph/0212020] [SPIRES].

[21] M.S. Carena and H.E. Haber, Higgs boson theory and phenomenology, Prog. Part. Nucl. Phys. 50 (2003) 63 [hep-ph/0208209] [SPIRES].

[22] J.A. Casas, A. Lleyda and C. Muñoz, Strong constraints on the parameter space of the MSSM from charge and color breaking minima, Nucl. Phys. B 471 (1996) 3 [hep-ph/9507294] [SPIRES].

[23] J.R. Ellis, J. Giedt, O. Lebedev, K. Olive and M. Srednicki, Against tachyophobia, Phys. Rev. D 78 (2008) 075006 [arXiv:0806.3648] [SPIRES].

[24] J.L. Evans, D.E. Morrissey and J.D. Wells, Vacuum stability with tachyonic boundary Higgs masses in no-scale supersymmetry or gaugino mediation, Phys. Rev. D 80 (2009) 095011 [arXiv: 0812.3874] [SPIRES].

[25] G. Bélanger, F. Boudjema, A. Pukhov and R.K. Singh, Constraining the MSSM with universal gaugino masses and implication for searches at the LHC, JHEP 11 (2009) 026 [arXiv:0906.5048] [SPIRES].

[26] B.C. Allanach, Naturalness priors and fits to the constrained minimal supersymmetric standard model, Phys. Lett. B 635 (2006) 123 [hep-ph/0601089] [SPIRES].

[27] R.R. de Austri, R. Trotta and L. Roszkowski, A Markov chain Monte Carlo analysis of the CMSSM, JHEP 05 (2006) 002 [hep-ph/0602028] [SPIRES].

[28] B.C. Allanach, K. Cranmer, C.G. Lester and A.M. Weber, Natural priors, CMSSM fits and LHC weather forecasts, JHEP 08 (2007) 023 [arXiv:0705.0487] [SPIRES].

[29] R. Trotta, F. Feroz, M.P. Hobson, L. Roszkowski and R. Ruiz de Austri, The impact of priors and observables on parameter inferences in the constrained MSSM, JHEP 12 (2008) 024 [arXiv:0809.3792] [SPIRES].

[30] Joint LEP2 SUSY Working Group, ALEPH, DELPHI, L3 and OPAL experiments homepage, http://lepsusy.web.cern.ch/lepsusy/.

[31] ALEPH collaboration, S. Schael et al., Search for neutral MSSM Higgs bosons at LEP, Eur. Phys. J. C 47 (2006) 547 [hep-ex/0602042] [SPIRES].

[32] Tevatron Electroweak Working Group collaboration, Combination of CDF and D0 results on the mass of the top quark, arXiv:0903.2503 [SPIRES].

[33] Particle Data Group collaboration, C. Amsler et al., Review of particle physics, Phys. Lett. B 667 (2008) 1 [SPIRES]. 
[34] Heavy Flavor Averaging Group collaboration, E. Barberio et al., Averages of b-hadron and c-hadron properties at the end of 2007, arXiv:0808.1297 [SPIRES].

[35] M. Misiak et al., The first estimate of $B R\left(B \rightarrow X_{s} \gamma\right)$ at $O\left(\alpha_{s}^{2}\right)$, Phys. Rev. Lett. 98 (2007) 022002 [hep-ph/0609232] [SPIRES].

[36] CDF collaboration, T. Aaltonen et al., Search for $B_{s}^{0} \rightarrow \mu^{+} \mu^{-}$and $B_{d}^{0} \rightarrow \mu^{+} \mu^{-}$decays with $2 \mathrm{fb}^{-1}$ of p p collisions, Phys. Rev. Lett. 100 (2008) 101802 [arXiv:0712.1708] [SPIRES].

[37] Z. Zhang, Muon g-2: a mini review, arXiv:0801.4905 [SPIRES].

[38] WMAP collaboration, E. Komatsu et al., Five-year Wilkinson Microwave Anisotropy Probe (WMAP) observations: cosmological interpretation, Astrophys. J. Suppl. 180 (2009) 330 [arXiv:0803.0547] [SPIRES].

[39] G. Bélanger, F. Boudjema, A. Pukhov and A. Semenov, MicrOMEGAs2.0: a program to calculate the relic density of dark matter in a generic model, Comput. Phys. Commun. 176 (2007) 367 [hep-ph/0607059] [SPIRES].

[40] G. Bélanger, F. Boudjema, A. Pukhov and A. Semenov, Dark matter direct detection rate in a generic model with MicrOMEGAs2.1, Comput. Phys. Commun. 180 (2009) 747 [arXiv:0803.2360] [SPIRES].

[41] N. Jarosik et al., Seven-year Wilkinson Microwave Anisotropy Probe (WMAP) observations: sky maps, systematic errors and basic results, arXiv: 1001.4744 [SPIRES].

[42] E. Komatsu et al., Seven-year Wilkinson Microwave Anisotropy Probe (WMAP) observations: cosmological interpretation, arXiv:1001.4538 [SPIRES].

[43] R. Barbieri and G.F. Giudice, Upper bounds on supersymmetric particle masses, Nucl. Phys. B 306 (1988) 63 [SPIRES].

[44] G. Jungman, M. Kamionkowski and K. Griest, Supersymmetric dark matter, Phys. Rept. 267 (1996) 195 [hep-ph/9506380] [SPIRES].

[45] D. Martí and A. Pomarol, Supersymmetric theories with compact extra dimensions in $N=1$ superfields, Phys. Rev. D 64 (2001) 105025 [hep-th/0106256] [SPIRES].

[46] K.-W. Choi, D.Y. Kim, I.-W. Kim and T. Kobayashi, SUSY flavor problem and warped geometry, hep-ph/0301131 [SPIRES].

[47] K.-W. Choi, D.Y. Kim, I.-W. Kim and T. Kobayashi, Supersymmetry breaking in warped geometry, Eur. Phys. J. C 35 (2004) 267 [hep-ph/0305024] [SPIRES].

[48] F. Brümmer, S. Fichet and S. Kraml, in preparation.

[49] The CDMS-II collaboration, Z. Ahmed et al., Results from the final exposure of the CDMS II experiment, arXiv:0912.3592 [SPIRES]. 